\title{
Deregulation of brain endothelial CD2AP in Alzheimer's disease impairs Reelin-mediated neurovascular coupling
}

\author{
Milène Vandal ${ }^{1}$, Colin Gunn ${ }^{1}$, Adam Institoris ${ }^{2}$, Philippe Bourassa ${ }^{3,4}$, Ramesh C. Mishra ${ }^{2}$, \\ Govind Peringod ${ }^{2}$, Camille Belzil ${ }^{1}$, Yulan Jiang ${ }^{1}$, Louise Reveret ${ }^{3,4}$, Cyntia Tremblay ${ }^{4}$, Mada \\ Hashem ${ }^{5}$, Wilten Nicola ${ }^{6}$, Jakob Körbelin ${ }^{7}$, Jeff F. Dunn ${ }^{1,2,5}$, Andrew P. Braun ${ }^{2}$, David A. \\ Bennett $^{8}$, Grant R.J. Gordon ${ }^{2}$, Frédéric Calon ${ }^{3,4 *}$, Minh Dang Nguyen ${ }^{1 *}$.
}

${ }^{1}$ Departments of Clinical Neurosciences, Cell Biology and Anatomy, and Biochemistry and Molecular Biology, Hotchkiss Brain Institute, University of Calgary, Calgary, Alberta, Canada. ${ }^{2}$ Department of Physiology and Pharmacology, Hotchkiss Brain Institute, Cumming School of Medicine, University of Calgary, Calgary, Alberta, Canada.

${ }^{3}$ Faculté de pharmacie, Université Laval, Québec, Québec, Canada.

${ }^{4}$ Axe Neurosciences, Centre de recherche du centre Hospitalier de l'Université Laval (CHUL), Québec, Québec, Canada.

${ }^{5}$ Department of Radiology, Hotchkiss Brain Institute, University of Calgary, Calgary, Alberta, Canada.

${ }^{6}$ Departments of Cell Biology and Anatomy, Hotchkiss Brain Institute, University of Calgary, Calgary, Alberta, Canada.

${ }^{7}$ Department of Oncology, Hematology and Bone Marrow Transplantation, University Medical Center Hamburg-Eppendorf, Germany.

${ }^{8}$ Rush Alzheimer's disease Center, Rush University Medical Center, Chicago, IL, United States.

Correspondence should be addressed to:

Drs. Minh Dang Nguyen, 3330 Hospital Drive NW, Calgary, Alberta T2N 4N1, Canada. E-mail: mdnguyen@ucalgary.ca and Frédéric Calon, 2705, boulevard Laurier, T-2-50, Québec, Québec, G1V 4G2, Canada. E-mail: frederic.calon@crchudequebec.ulaval.ca

Short title: Brain vascular CD2AP in Alzheimer's disease

Key words: Alzheimer, cerebrovascular function, neurovascular coupling, brain perfusion, brain endothelial cells, CD2AP, Reelin, ApoER2 / LRP8. 


\begin{abstract}
Cerebrovascular dysfunction is increasingly recognized as a major contributor to Alzheimer's disease (AD). CD2-associated protein (CD2AP), an important predisposing factor for the disease, is enriched in the brain endothelium but the function of protein in the brain vasculature remains undefined. Here, we report that lower levels of CD2AP in brain vessels of human AD volunteers are associated with cognitive deficits. In awake mice, we show that brain endothelial CD2AP regulates cerebral blood flow during resting state and functional hyperemia. In the endothelium, CD2AP controls the levels and signaling of apolipoprotein E receptor 2 (ApoER2), a receptor activated by Reelin glycoprotein that is linked to memory function. Further, Reelin promotes brain vessel dilation and functional hyperemia and both effects are modulated by endothelial CD2AP. Finally, lower levels of ApoER2 in brain vessels are associated with vascular defects and cognitive dysfunction in AD individuals. Thus, deregulation of CD2AP impairs neurovascular coupling and harnessing the biology of the Reelin-ApoER2-CD2AP signaling axis in the brain endothelium may improve brain vascular dysfunction in AD patients.
\end{abstract}




\section{Introduction}

Alzheimer's disease (AD) is the leading cause of dementia worldwide ${ }^{1}$. Brain hypoperfusion ${ }^{2,3}$ and blood brain barrier (BBB) defects ${ }^{4-6}$ are amongst the earliest events observed in the disease ${ }^{7}$. They precede the accumulation of plaques and tangles ${ }^{4,5,8}$ and are tightly linked to the neuropathological and clinical manifestations of the disease ${ }^{9-14}$. Deterioration of the brain endothelium is also observed in AD patients and mouse models ${ }^{5,15-17}$. The mechanisms underlying brain endothelial cells (BECs) dysfunction in $A D$ and their contribution to disease pathogenesis remains however poorly understood.

Polymorphisms in CD2-associated protein (CD2AP), a scaffolding protein that controls cytoskeletal remodeling and membrane trafficking during receptor endocytosis and cell-cell interactions ${ }^{18,19}$, represent one of the top 10 genetic predisposition factors for AD (alzgene.org, $\left.{ }^{20}\right)^{21-24} 25$. Because CD2AP is involved in endocytic trafficking ${ }^{26}$, an important step in the generation of $A \beta^{27}$, several studies have proposed a role for the protein in endocytic $A \beta$ production in neurons ${ }^{28-30}$. This function corroborates the association between CD2AP polymorphism and increased neuritic plaque accumulation in AD patients ${ }^{31}$. However, CD2AP haploinsufficiency has limited impact on $A \beta$ accumulation in a mouse model of $A D{ }^{29}$, raising some doubt as to whether endocytic $A \beta$ production from brain cells is the defining role of CD2AP in $A D$.

Interestingly, endothelial cell degeneration is observed in the glomerulus of CD2AP KO mice ${ }^{32}$. Genetic variations in CD2AP are associated to fibromuscular dysplasia ${ }^{33}$ and brain hypometabolism in healthy volunteers ${ }^{34} . C D 2 A P$ is also discussed as candidate gene to account for reduced left angular gyrus cerebral blood flow (CBF) in participants from the Alzheimer's Disease Neuroimaging Initiative (ADNI) study ${ }^{35}$. Mouse tissue immunostaining ${ }^{36,37}$ 
and single-cell RNAseq ${ }^{38}$ revealed that CD2AP is highly enriched in brain endothelial cells (BECs). However, the role of CD2AP in the brain endothelium remains undefined.

Since brain vascular dysfunction impacts $A D$ progression ${ }^{2}$, we postulated that CD2AP may have an uncharacterized function in brain vessels that once altered, could contribute to $A D$ pathogenesis.

\section{Results}

\section{Brain vascular CD2AP is associated with cognitive function in AD volunteers}

To gain insights into the role of CD2AP in AD pathogenesis, we first analyzed the levels of CD2AP in homogenates containing detergent-soluble proteins isolated from parietal neocortices of subjects from the Religious Order Study, a thoroughly characterized longitudinal clinicalpathologic study of aging and dementia ${ }^{39,40}$. Sex, cognitive performance and neuropathology for all participants are available in Extended data Table 1 and were previously published ${ }^{15,41}$. No difference was found for the levels of CD2AP in cortical extracts containing all brain cells when participants were classified according to their neuropathological scores (Extended data Figure $1 A$ ) or clinical diagnosis (Figure $1 A-B$ ). In line with previous observations made in cell cultures ${ }^{30}$ and Drosophila melanogaster ${ }^{42}$, we found that the levels of CD2AP inversely correlate with the amount of soluble $A \beta 42$ (Figure 1C), A $\beta 40$ (Figure 1D) and insoluble tau accumulation in the human parietal cortex (Figure 1E) (Extended data Table 2). We next assessed protein expression in brain vascular samples containing a mix of brain arterioles, arteries and capillaries (see ${ }^{15}$ for a complete description of the content) prepared from parietal cortices of the same cohort of volunteers. CD2AP protein was found enriched in these human brain vessel preparations (Figure 1F and G), similar to findings in mouse brain tissue ${ }^{36,37,43}$. Interestingly, when participants were classified based on clinical diagnosis, the levels of vascular CD2AP were reduced by $\sim 30 \%$ in the brain of $A D$ subjects when compared with $\mathrm{NCl}$ volunteers (Figure $1 \mathrm{H}$ and I and Extended data Figure 1B). There was no change in the levels of the endothelial cell 
marker CD31 (Extended Figure $1 \mathrm{C}$ and ${ }^{41}$ ), suggesting that the reduction in CD2AP is not associated with endothelial cell death. Remarkably, the lower levels of CD2AP in the brain vascular fraction correlated with cognitive dysfunction, as indicated by several cognitive parameters including the last MMSE score and global cognitive score (Figure $1 \mathrm{~J}$ and Extended data Table 2). Thus, reduced levels of CD2AP in brain vessels are associated with cognitive dysfunction in AD volunteers.

\section{Brain endothelial CD2AP regulates functional hyperemia and cerebral blood flow}

CD2AP KO mice die around 6 weeks of age from renal failure ${ }^{44}$ and a podocyte-specific CD2AP overexpression rescues the early lethality of these mice ${ }^{45}$. However, these mutant compound mice still develop multiple phenotypes including BBB leakage and seizures ${ }^{46,47}$, thereby making them unsuitable to study the physiological function of CD2AP in brain vasculature. To circumvent this issue, we generated 3 adeno-associated viruses (AAV-BR1-GFP) specifically targeting the BECs ${ }^{48}$. The viral vectors contained short hairpin RNAs (shRNAs) directed against CD2AP mRNA or a random non-specific sequence (Figure 1L). Specificity of the CD2AP siRNA was tested in cell cultures and consistently, caused a $70-80 \%$ decrease of CD2AP in primary mouse BECs and in a human BEC line (Extended data Figure 2). The viral vectors were injected retro-orbitally in 30-day-old mice (Figure $1 \mathrm{M}$ ). After three weeks, a timeline known for the viral vector to be expressed ${ }^{48}$, AAV-BR1-GFP-CD2AP shRNA (termed AAV-CD2AP) viral vector produced a subtle, yet significant knockdown of CD2AP in the cortex, as demonstrated by western blots, when compared to mice injected with the control viral vector (AAV-Control) (Figure $1 \mathrm{~N}$ and $\mathrm{O}$ ). The viral vector infected BECs lining the vessels, as visualized with twophoton microscopy in mice i.v. injected with rhodamine-dextran to highlight the lumen (Figure 1P). Because lower levels of vascular CD2AP are linked to cognitive dysfunction in AD volunteers (Figure 1J), we first asked the question whether CD2AP is important for functional hyperemia i.e. the process by which cerebral blood flow (CBF) rapidly adjusts to fulfill the local 
energy demands of increased neuronal activity ${ }^{49}$. This mechanism of coupling between the neuronal and the vascular systems is impaired in AD patients ${ }^{50,51}$ and correlates with performance in cognitive tasks ${ }^{49,52}$. We evaluated functional hyperemia in the barrel cortex of awake mice trained for head fixation on a passive, air-supported spherical treadmill (Figure 1M) (For detailed methods see ${ }^{53}$ ). Briefly, pial vessels lumen area was calculated using a newly developed semi-automated algorithm (Extended data video 1), before and after a 5 sec sensory stimulation of facial whisker using an air puff (Figure 1Q). Interestingly, the maximal vessel dilation in response to whisker stimulation was reduced by $\sim 50 \%$ in mice with BECs depleted of CD2AP when compared to-that of control mice (Figure 1R). Next, since CD2AP has been suggested as a candidate gene associated with lower resting CBF in AD patients ${ }^{35}$, we investigated the effect of lowering CD2AP in BECs on resting CBF using arterial spin labelling magnetic resonance imaging (MRI). We performed MRI two and three weeks after viral injection (Figure $1 \mathrm{M}$ ) and found that mice with knockdown of CD2AP in BECs displayed a $5 \%$ reduction in resting cortical $\mathrm{CBF}$, three weeks after viral injection when compared to the same animals analyzed one week earlier (Figure 1S to U). Such difference was not observed between the second and third week in the cortex of mice injected with the control virus (Figure 1T).

Furthermore, we found a positive correlation between cortical CBF and CD2AP levels evaluated by western blot: the lower the levels of CD2AP, the lower resting CBF (Figure 1V). Thus, CD2AP depletion in BECs in vivo reduces basal CBF and impairs neurovascular coupling.

\section{CD2AP controls ApoER2 levels and Reelin-mediated signaling in BECs}

Apolipoprotein $\mathrm{E} 4$ (APOE4), the main genetic risk factor for late onset $\mathrm{AD}$, is involved in most aspects of $A D$ pathogenesis, including vascular dysfunction ${ }^{54} . C D 2 A P$ polymorphisms are linked to the presentation of $A D$ symptoms in $A P O E 4$ carriers ${ }^{55}$ and to a loss of association between plaques and plasma ApoE ${ }^{56}$. The levels of $\mathrm{ApoE}$ are determined by binding to and trafficking of the Low Density Lipoprotein (LDL) receptor family that includes Apolipoprotein $\mathrm{E}$ 
receptor 2 (ApoER2) ${ }^{57}$. Interestingly, the trafficking of ApoER2 that participates in ApoE endosomal recycling ${ }^{57}$, is regulated by CD2AP homologue Cbl interacting protein $85(\mathrm{CIN85})^{58}$. Therefore, we determined whether ApoER2 is a potential target for CD2AP in the brain endothelium. We first confirmed the enrichment of CD2AP in BECs using immunostaining of cultured mouse primary BECs and western blot of lysates derived from these cells (Figure 2A, Extended data Figure 3). Next, using a human BEC line (hCMEC/D3) and mouse brain tissues, we tested the putative association between CD2AP and ApoER2. We found that ApoER2 coimmunoprecipitates with CD2AP (Figure 2B and C) and this association was detectable in different buffers with various concentrations of ionic and non-ionic detergents (Figure 2D). Dynamin 2, a key protein for receptor endocytosis and membrane trafficking, was also detected in CD2AP immunoprecipitates (Figure 2D), supporting a role for CD2AP in ApoER2 trafficking. No interaction was found between CD2AP and PICALM (Phosphatidylinositol Binding Clathrin Assembly Protein, see Figure 2D), another endocytic molecule associated with increased risk for $A D^{24}$. In brain lysates from adult mice, CD2AP antibodies immunoprecipitated both CD2AP and ApoER2 (Figure 2C). The reverse co-immunoprecipitation using ApoER2 antibodies also pulled down CD2AP (Figure 2C). To understand the role of CD2AP on ApoER2 function, we silenced the expression of CD2AP in mouse and human BECs using siRNA. Knockdown of CD2AP had no effect on the levels of Dynamin 2 and PICALM (Figure 2E and F) but caused a significant $\sim 40 \%$ decrease in levels of ApoER2 and VE-Cadherin (Figure 2E and F), the data on VECadherin being consistent with the increased BBB permeability found in CD2AP compound mutant mice ${ }^{46}$. Similar results were obtained with 2 different siRNAs in both human and mouse BECs, thereby providing specificity to the observed phenotypes (Figure 2E and F). We then explored how CD2AP regulates the levels of ApoER2. Specifically, the misprocessing of ApoER2 in CD2AP-depleted cells was rescued with an active form of the Rab5 GTPase (Figure $2 \mathrm{G}$ and $\mathrm{H}$ ) that is involved in membrane trafficking ${ }^{59}$, or inhibition of $\gamma$-secretase (Figure $2 \mathrm{~J}$ and K) that cleaves ApoER2 to release a C-terminal fragment ${ }^{60}$. Our results on the effects of CD2AP 
loss function on ApoER2 levels are reminiscent of those showing that ApoE4 also impairs the trafficking of ApoER2 resulting in its degradation ${ }^{57}$; they are also in agreement with observations that processing of the receptor is disturbed in the cortex of AD patients ${ }^{61,62}$. Last, to determine whether ApoER2 signaling is impaired in the absence of CD2AP, we treated CD2AP-depleted cells with the glycoprotein Reelin, a well characterized ligand for ApoER2, secreted by astrocytes and neurons with protective effects against parenchymal $A \beta$ toxicity in animal models of $A D^{63,64}$. The canonical Reelin signaling pathway involves the binding of Reelin to ApoER2 and this leads to the phosphorylation of the adaptor protein disabled-1 (Dab1), and subsequently to the phosphorylation/activation of protein kinase B (Akt) at Ser473 ${ }^{65}$. This signaling is dampened by Dab2, an adaptor protein that strongly associates with CD2AP ${ }^{66}$ and ApoER2 ${ }^{67,68}$, and is involved in the recycling of the receptor through inactivation of Akt ${ }^{69}$. BECs transfected with control siRNA and treated with Reelin for 30 and 120 min undergo signaling via the canonical pathway. At both time points, these cells showed activation of Akt (measured by the p-Ser 473 Akt/total Akt ratio) with an attenuation at 120 min (Figure 2L), and displayed a $\sim 30 \%$ increase in levels of ApoER2 (Figure 2L). In contrast, CD2AP-depleted cells treated with Reelin displayed at both time points an hyperactivation of the kinase, known to interfere with ApoER2 recycling ${ }^{69,70}$, and consistently, lower levels of the receptor (Figure $2 \mathrm{~L}$ ). Taken together, our data revealed that CD2AP is in complex with ApoER2, and regulates both the levels and Reelin-dependent signaling of the receptor in BECs.

\section{Reelin effect on neurovascular coupling is mediated via the endothelium in a nitric oxide synthase-dependent manner}

Reelin is well characterized for its role in synaptic plasticity in the mature brain ${ }^{71-75}$, and promotes angiogenesis in the developing brain ${ }^{76}$. Interestingly, the glycoprotein is secreted from liver stellate cells into the bloodstream ${ }^{77,78}$ and expressed in BECs ${ }^{79}$. However, the role of Reelin in the adult brain vasculature remains unknown. We therefore investigated the putative 
effects of purified Reelin on brain vascular tone and neurovascular coupling in awake mice. The brain microvasculature was visualized after IV injection of fluorescein-isothianate dextran (2000 $\mathrm{kDa}$ ) (Figure $3 \mathrm{~A}$ and $\mathrm{B}$ ). A cover glass with holes was applied on the craniotomy site to allow the superfusion of Reelin $(1 \mu \mathrm{M})$ or control solution (see ${ }^{72}$ for a detailed description of the solutions) on the brain surface during the recordings (Figure 3B). Lumen cross-sectional area was recorded for 5 min before the perfusion of control solution or Reelin that lasted for 20 min (Figure $3 C)$. Remarkably, Reelin consistently caused a robust $\sim 80 \%$ dilation of penetrating arterioles (Figure 3D to G). We next assessed the effects of Reelin on functional hyperemia using 5 sec of whisker stimulation (Figure $3 \mathrm{H}$ and I). Notably, Reelin treatment increased peak dilation by $70 \%$ (Figure 3J-K) and the overall vascular response by 150\% (Figure 3L) during functional hyperemia.

To determine whether the vasodilatory effect of Reelin was mediated through the endothelium and/or mural cells of the neurovascular unit, we next performed pressure myography analysis of cerebral arteries (passive diameter $146 \pm 15 \mu \mathrm{m}, \mathrm{n}=12$ ) freshly isolated from mouse brains (Figure 3M), The arteries were maintained at the pressure of $70 \mathrm{mmHg}$ and tested with vasoconstrictive (i.e. phenylephrine, $250 \mathrm{nM}$ ) and vasodilatory drugs (i.e. KCa channel activator SKA-31 ${ }^{80}(3$ and $15 \mu \mathrm{M})$, bradykinin $(0.5 \mu \mathrm{M})$, sodium nitroprusside (SNP $\left.10 \mu \mathrm{M}\right)$, Pinacidil (10 $\mu \mathrm{M})$ ) to confirm their overall health and functionality before and after the application of Reelin or control solution (Figure 3N, O and Extended data Figure 4A). Bath application of Reelin (1 $\mu \mathrm{M}$ ) consistently promoted vasodilation of small arteries whereas the control solution had no effect (Figure $3 \mathrm{~N}$ and $\mathrm{P}$ ). To isolate the effects of Reelin on BECs, we mechanically disrupted the endothelial layer by injecting an air bubble in the lumen, washed the debris out and subjected the endothelium-denuded arteries to the same drug treatments. In the absence of the endothelium (confirmed by loss of SKA-31 and bradykinin-evoked dilation in endotheliumstripped arteries; Extended data Figure $4 \mathrm{~A},{ }^{81}$ ), the vasodilatory effect of Reelin was reduced by 
$\sim 70 \%$ (Figure 30 and $\mathrm{P}$ ). This finding demonstrates that receptors expressed by the brain endothelium are important for the vasodilatory effect of Reelin.

Endothelial nitric oxide (NO) can modulate the arteriole response to whisker stimulation ${ }^{82,83}$. Since activation of ApoER2 activates endothelial nitric oxide synthase (eNOS) ${ }^{84}$ to produce NO, we next asked the question whether the effect of Reelin is dependent on eNOS. We blocked NOS with the non-selective inhibitor N5-(1-Iminoethyl)-L-ornithine dihydrochloride (L-NIO, a more efficient inhibitor for eNOS than neuronal NOS ${ }^{85}$ ), in awake mice subjected to two-photon imaging. L-NIO (300 $\mu \mathrm{M})$ was superfused on the brain surface for 20 min to block NOS before starting the Reelin $(1 \mu \mathrm{M})$ superfusion that lasted for 20 min (Figure 3Q, Extended data Figure 4B). Consistent with previous observations, L-NIO decreased the lumen cross-sectional area ${ }^{53}$ (Extended data Figure 4D). Importantly, in presence of L-NIO, Reelin did not increase the lumen cross-sectional area when compared to treatment with L-NIO alone (Extended data Figure 4E). Next, to determine whether the effect of Reelin on functional hyperemia was dependent on NOS signaling, we evaluated dilation of penetrating arterioles in response to whisker stimulation before L-NIO as well as before and after Reelin + L-NIO (Figure 3R). We found that the peak dilation in response to whisker stimulation was reduced by $\sim 30 \%$ after Reelin application in the

presence of L-NIO, when compared to the peak dilation before L-NIO (Figure 3S). Similarly, the overall response was reduced by $\sim 40 \%$ following Reelin + L-NIO compared to L-NIO alone (Figure $3 \mathrm{U})$. In sum, the in vivo vasodilatory action of Reelin on brain vessels depends on NO and occurs largely through the endothelium.

\section{CD2AP modulates the neurovascular effects of Reelin in vivo}

In cultured BECs, CD2AP regulates the Reelin-mediated ApoER2 signaling (Figure 2). To determine whether CD2AP modulates the cerebrovascular effects of Reelin in vivo, we superfused Reelin for 20 min on the brain surface of awake AAV-CD2AP and AAV-Controlinjected mice during two-photon imaging. In AAV-control animals, Reelin showed a more limited 
effect on vessel dilation (Figure 3V and W) than in non-injected animals (Figure 3D), possibly because BECs are highly sensitive to stress ${ }^{86}$ and their cellular response to Reelin might be modified upon AAV infection. Nevertheless, Reelin caused a modest vessel dilation in AAVCD2AP animals compared to AAV-control animals (Figure 3V-W). Clearer still, Reelin increased the overall response to whisker stimulation in AAV-CD2AP-injected mice (Figure 3Y), consistent with the overactivation of Akt found in CD2AP-depleted cells that lies upstream of eNOS required for the effect of Reelin on brain vessel dilation. However, the peak dilation during functional hyperemia after Reelin superfusion was still 30\% lower in AAV-CD2AP compared to AAV-Control animals (Figure 4Z). These results suggest that loss of functional CD2AP in BECs deregulates the response of brain vessels to Reelin.

\section{Lower levels of ApoER2 in brain vessels correlate with reduction of vascular CD2AP, cognitive dysfunction and vascular pathology in AD volunteers}

Since Reelin-mediated ApoER2/CD2AP signaling impacts brain vascular function (Figure 1 and 3), we next studied the associations between ApoER2 and the neuropathological and clinical manifestations of AD. After confirming that ApoER2 is present in brain vessels by immunostaining (Figure 4A), we quantified by western blots the levels of ApoER2 in brain vascular preparations from volunteers of the Religious Order Study (the levels of ApoER2 in the parietal cortex are also available in Extended data Figure $5 \mathrm{~A}$ to $\mathrm{C}$ and Extended data Table 2). Upon classification according to the clinical diagnosis, we found that the levels of ApoER2 in brain vessels are reduced by $\sim 60 \%$ in $\mathrm{AD}$ compared to $\mathrm{NCl}$ participants (Figure $4 \mathrm{~B}$ and $\mathrm{C}$ ). In contrast, ApoER2 concentrations were not associated with the neuropathological diagnosis (Extended data Figure 5D). Similarly to our cell culture results that show a CD2AP-dependent regulation of ApoER2 levels (Figure 2), we observed a significant association between the levels of CD2AP and those of ApoER2 in these brain vessel extracts (Figure 4D). Consistent with the observation that ApoER2 levels are downregulated by ApoE4 ${ }^{57}$, we also detected lower levels of ApoER2 in 
APOE4 carriers (Figure 4E). Remarkably, the lower levels of brain vascular ApoER2 correlated with ante mortem cognitive dysfunction in AD subjects, as assessed with the last MMSE score and global cognitive score, including semantic memory, perceptual speed and episodic memory (Figure 4F and Extended data Table 2). Associations between ApoER2 and CD2AP and cognitive dysfunction remained significant after adjustment for plaques and tangle counts (Figure 4F and Extended data Table 2), suggesting that they are independent of classical AD neuropathology. Overall, these data indicate that a reduction in brain vascular ApoER2 is associated with cognitive decline.

Next, we looked at the relationship between ApoER2 and CD2AP and cerebrovascular dysfunction. Mural cells are involved in the regulation of $\mathrm{CBF}^{87}$ and maintenance of $\mathrm{BBB}{ }^{88}$, and their dysfunction is related to AD pathogenesis (see refs ${ }^{8,12,41,89}$ by our group and others). Here, we found that CD2AP and ApoER2 concentrations in vascular extracts were lower in subjects with low levels of the mural cell markers Platelet-derived growth factor receptor beta (PDGFR $\beta$ ) (Figure $4 \mathrm{G}$ and $4 \mathrm{H}$ ) and alpha-smooth muscle actin ( $\alpha-S M A)$ (Figure $4 \mathrm{I}$ and $4 \mathrm{~J}$ ), that were both reported to be decreased in the brain of $A D$ volunteers ${ }^{41}$. Cerebral amyloid angiopathy (CAA) and cortical infarcts are also frequently observed in $A D{ }^{90,91}$. In this regard, we observed that ApoER2 levels were $\sim 60 \%$ lower in individuals with cortical infarcts, while such difference was not observed for CD2AP (Figure 4K and L). Finally, the levels of vascular ApoER2 (but not vascular CD2AP levels) were inversely correlated to the concentration of $A \beta 40$ and $A \beta 42$ in the same isolated brain vessel extracts (Figure 4M to P). Taken all together, our findings suggest that defects in brain vascular CD2AP and ApoER2 contribute to cognitive decline and AD pathogenesis. 


\section{Discussion}

Within the neurovascular unit, pericytes work in close relationship with BECs to maintain CBF and BBB integrity ${ }^{88,92,93}$ and accumulating evidence suggests that they may be critical for the early vascular deficits observed in $A D^{3,8,94,95}$. Although brain endothelium defects have been characterized in $A D^{5,10,15,96,97}$, the molecular mechanisms underlying $B E C$ dysfunction remain poorly understood. In this study, we addressed this fundamental question by identifying a new signaling axis in the cerebral endothelium tied to the control of CBF and to the clinical manifestations of the disease. We show that lowering CD2AP in BECs impairs functional hyperemia and reduces basal CBF in awake mice. Furthermore, Reelin glycoprotein, a well characterized ligand for ApoER2, causes a robust, nitric oxide-dependent vasodilation of penetrating arterioles and functional hyperemia. These in vivo effects of Reelin are both deregulated in mice with brain vessels depleted of CD2AP, in line with an overshoot in Reelin signaling observed in BECs lacking CD2AP.

In the adult brain, Reelin has been mostly characterized as a mediator of synaptic plasticity and plays an important role in memory function ${ }^{71-75}$. The synaptic function of Reelin is antagonized by $A \beta^{98}$ and the protein can counteract tau and $A \beta$ pathologies ${ }^{63,64,99,100}$. The levels of the glycoprotein are also altered in the CSF and cortical tissues of AD patients ${ }^{101,102}$. Our study now reveals that Reelin is involved in the control of vessel tone in the basal state and in response to neuronal activation, two process altered in the brain of AD patients ${ }^{3,5,50}$, thereby expanding the implication of this glycoprotein in AD. Finally, the levels of both CD2AP and ApoER2 are decreased in brain vessels of $A D$ individuals and importantly, these reductions are associated with cognitive dysfunction as well as vascular defects in the case of ApoER2. Cumulatively, our results suggest that defects in the endothelial Reelin-ApoER2-CD2AP signaling axis cause vascular impairments that are relevant to $A D$. 
How the Reelin-ApoER2-CD2AP axis in the brain endothelium interface with other pathogenic mechanisms in $A D$ remain to be fully investigated. Our study indicates that lower vascular levels of CD2AP are linked to cognitive dysfunction but not to $A \beta$ and tau, suggesting that this association is explained by another mechanism. We advance a novel function for CD2AP in the regulation of neurovascular coupling and resting blood flow through BECs and in response to Reelin. Interestingly, functional hyperemia, is tightly linked to cognitive function ${ }^{103,104}$ and has been consistently reported to be altered at different stages of $A D{ }^{51}$. Change in functional hyperemia in $A D$ is also linked to brain hypoperfusion ${ }^{105}$. Therefore, loss of CD2AP function might impair neurovascular coupling in $A D$ and this would explain the association between the lower levels of CD2AP in brain vessels and cognitive dysfunction in AD patients.

Endothelial CD2AP deregulation likely contributes to cerebrovascular dysfunction through cell autonomous and non-cell autonomous mechanisms. For instance, we observed an association between cortical CD2AP and $A \beta$. A $\beta$ produced by neurons constricts capillaries through pericytes via generation of reactive oxygen species and endothelin-1 signaling ${ }^{87}$. Increased endothelin-1 expression is observed in CD2AP KO mice ${ }^{106}$ and therefore, the loss of functional CD2AP could contribute to the constriction of cerebral vessels via endothelin-1 overexpression and $\beta$-amyloid accumulation. Since CD2AP regulates trafficking of PDGFR ${ }^{26}$, decreased levels of vascular CD2AP in patients with low levels of $\alpha$-SMA and PDGFR $\beta$ (Fig.4) further suggest an intimate interplay between mural cells health and vascular CD2AP. In this model, increased A $\beta$ generation and endothelin-1 combined with reduced levels of PDGFR $\beta$ and disrupted cell-cell interactions at the BBB (as found in CD2AP KO ${ }^{46}$ and BECs depleted of CD2AP - see the reduced levels of VE-Cadherin in Fig.2) could exacerbate mural cells degeneration and increase BBB permeability, a phenomenon that is observed in the preclinical stages of $A D^{8}$ and in patients with the APOE4 genotype ${ }^{12}$. Alterations in ApoER2 consequential to the loss of function 
of CD2AP and/or to the APOE4 genotype ${ }^{57}$ would have an additive effect on these pathogenic processes. We also found an inverse correlation between the load of $A \beta$ in cortical tissues and brain vessels, and ApoER2 levels. Interestingly, CAA that is characterized by the accumulation $A \beta$ in brain vessels, is present in both $A D$ patients and $A P O E 4$ carriers and associated with changes in $A \beta$ clearance ${ }^{91}$. Perhaps, the association between vascular ApoER2 and vascular $A \beta$ reflects a role for ApoER2 in A $\beta$ clearance, a function fulfilled by LDR receptors ${ }^{16,54}$. Increasing $A \beta$ load deregulates Reelin-mediated ApoER2 signaling ${ }^{98}$ during synaptic plasticity ${ }^{71-75}$ but this might also alter Reelin's function on brain vessel dilation and functional hyperemia, thereby affecting memory function via multiple mechanisms.

In humans, genetic changes in $C D 2 A P$ are associated with both focal segmental glomerulosclerosis, a leading cause of proteinuric kidney disease that progresses to end-stage renal failure ${ }^{107}$ and to $A D^{20}$. The brain and the kidney are both densely vascularized organs very vulnerable to small vessel dysfunction ${ }^{108,109}$. The two main causes of chronic kidney disease are diabetes and high blood pressure ${ }^{110}$, and both are key risk factors for AD ${ }^{111}$. Further, chronic kidney disease also predicts cognitive decline ${ }^{112}$. Given the role of CD2AP on vascular health unveiled here, it is logical that its dysfunction will compromise both organs. Accordingly, genes linked to the kidney system are emerging as risk factors for AD ${ }^{113}$ while patients with chronic kidney disease are predisposed to cognitive dysfunction and dementia ${ }^{109,114,115}$. In summary, our data suggest that the Reelin-ApoER2-CD2AP axis may provide an upstream therapeutic target to prevent vascular pathology and consequent symptoms in AD. Developing therapeutic strategies around the biology of this axis or vascular / blood-borne factor-elicited signaling pathways to control neurovascular coupling and CBF may open a new era of research in AD. 


\section{Methods}

\section{Animals}

Male C57BL6 mice between 4 and 8 weeks of age were maintained in a room kept at $22^{\circ} \mathrm{C}$ with a $12 \mathrm{~h}$ light cycle and had ad libitum access water and standard chow diet. All animal procedures were performed in accordance with the Canadian Council on Animal Care guidelines and were approved by the Health Sciences Animal Care Committee at the University of Calgary.

\section{Reelin purification}

Reelin was purified from conditioned media of HEK 293 cells stably transfected with Reelin cDNA (a gift from Dr. Joachim Herz laboratory, University of Texas Southwestern Medical Center, and Dr. Tom Curran, University of Pennsylvania) as per ${ }^{72,116-118}$. Briefly, for the cell culture experiments, cells were cultured in low glucose DMEM (Gibco, Thermo Fisher, Waltham, M. 11885084) supplemented with FBS until they reached full confluency and then, the media was switched to phenol red-free Opti-MEM for 2 days. Afterward, the conditioned Opti-MEM was centrifuged at $5000 \mathrm{~g}$ for $15 \mathrm{~min}$ to pellet any cell debris. The clarified media was then sterile filtered and concentrated by 30 times using Amicon Ultra-15 100 kDa Centrifugal Filter Units (EMD Millipore). For the pressure myography and the awake two-photon imaging experiments, after the cells reached full confluency, the media was switched to low glucose DMEM (Gibco, Thermo Fisher, Waltham, M. 11885084) supplemented with $0.01 \%$ bovine serum albumin (BSA) for 2 days. After, the media was centrifuged at $5000 \mathrm{~g}$ for $15 \mathrm{~min}$ to pellet any cell debris. The supernantant was concentrated 30 times using Amicon Ultra-15 100 kDa Centrifugal Filter Units (EMD Millipore). The media was exchanged to buffer $\mathrm{A}$ (20 mM Tris, $2 \mathrm{mM} \mathrm{CaCl} 2,10 \mathrm{mM} \mathrm{NaCl}$ ) and concentrated again 30 times. The concentrated media in buffer A was purified using GSepharose (Sigma) beads with buffer A from 0.1 M to 0.4 M NaCl concentration. The Reelin samples were collected between $0.25 \mathrm{M}$ and $0.3 \mathrm{M} \mathrm{NaCl}$. The samples were concentrated 30 
times using Amicon Ultra-15 100 kDa Centrifugal Filter Units (EMD Millipore). The solution was diluted in PBS and concentrated again using an Amicon Ultra-4 50 kDa Centrifugal Filter Unit (EMD Millipore) to replace the media with PBS while retaining the dissolved Reelin. The control solution was prepared with the same procedure used for the Reelin solution but instead of using Reelin-transfected HEK 293 cells, non-transfected HEK 293 cells were used. The concentration of Reelin was measured by DC Assay (BioRad Laboratories), whereas presence of the glycoprotein in the purified fraction was confirmed on Western blot with anti-Reelin antibodies, and was verified with mass spectrometry according to published methods ${ }^{72,117}$. Reelin activity was confirmed by Western blot using antibodies directed against phospho-Serine 473 Akt, a downstream target of the Reelin pathway, in cell lines, as well as by comparing with that of a commercially available central active fragment of Reelin.

\section{Awake two-photon imaging}

All procedure for awake two-photons imaging were performed according to a previously published protocol ${ }^{53,119}$. Briefly, two-photon imaging was performed on male C57BL6 aged between 6 and 8 weeks old to evaluate the effect of Reelin and L-NIO on vessel dilation and neurovascular coupling. Day 1, head bar installation: Mice were kept under anesthesia with isoflurane and received a sub-cutaneous injection of buprenorphine $(0.05 \mathrm{mg} / \mathrm{kg})$ and enrofloxacin $(2.5 \mathrm{mg} / \mathrm{kg})$. Under aseptic conditions, the skin on top of the head was removed and the skull was cleaned with lactate ringer before a metal head bar $(0.5 \mathrm{~g})$ was glued on the occipital bone using fast glue. The head bar position was secured on the brain using first 2 layers of a three components dental glue (C\&B Metabond, Parkell Inc, NY, USA) and dental cement (OrthoJet Acrylic Resin, Land Dental MFG. CO., Inc., IL, USA) was added to form a well large enough to receive the microscope objective. A cut $16 \mathrm{G}$ needle was inserted in the well to allow drug superfusion during the imaging session. Day 2 and 3 , training sessions: After $24 \mathrm{~h}$ of recovery, mice were habituated to run on an air-supported spherical Styrofoam ball. Mice were 
head-fixed and were allowed to run for 15 min after which, intermittent air stimulation was delivered to the contralateral whiskers (15 stimulations, $5 \mathrm{~s}$, every 60 s). Day 4 , craniotomy: Mice were anesthetized with isoflurane and received an injection of buprenorphine for pain control $(0.05 \mathrm{mg} / \mathrm{kg})$. The bone and the dura were removed gently over the barrel cortex with constant immersion under HEPES CSF (142 mM NaCl, 5 mM KCl, 10 mM Glucose, 10 mM HEPES, 3.1 $\mathrm{mM} \mathrm{CaCl}_{2}, 1.3 \mathrm{mM} \mathrm{MgCl}_{2}$ ). After the surgery was completed, the cranial window was sealed with a cover glass with nine $0.175 \mathrm{~mm}$ holes using fast glue. The well was constantly maintained under aCSF to prevent the brain surface from drying. For some of the experiments, animals received a $150 \mu$ l retro-orbital injection of 5\% FITC-dextran (MW 2,000,000, Sigma) dissolved in lactate ringer. The animals were then transferred to the microscope and imaging was started about 45 min later.

Drug application during awake two-photon imaging

The brain surface was superfused with aCSF at $0.9 \mathrm{ml} / \mathrm{min}$ with a carbogen pressurized system $\left(95 \% \mathrm{O}_{2}, 5 \% \mathrm{CO}_{2}\right)$. The drugs were pre-dissolved in aCSF and setup on a syringe pump adjusted at $0.1 \mathrm{ml} / \mathrm{min}$, allowing a 1 in 10 dilution of the drug applied on the brain surface. Functional hyperemia

Pial arteriole were first distinguished from venules by vasomotion and response to whisker stimulations. Either the pial vessels or the connected penetrating arterioles response to contralateral whisker stimulation was recorded (10 to 100 microns depth). An air puff (5 s) was delivered through two glass tubes to the contralateral whiskers after recording a $5 \mathrm{sec}$ baseline. Response to whisker stimulation was recorded three times before and after each drug was applied.

Two-Photon Fluorescence Microscopy

Imaging was performed using a custom built two-photon microscope ${ }^{120}$ fed by a Ti:Sapph laser source (Coherent Ultra II, $4 \mathrm{~W}$ average output at $800 \mathrm{~nm}, \sim 80 \mathrm{MHz}$ ). Image data were acquired using MatLab (2013) running an open source scanning microscope control software called 
Scanlmage (version 3.81, HHMI/Janelia Farms) ${ }^{121}$. Imaging was performed at an excitation wavelength of $800 \mathrm{~nm}$ for FITC-dextran or $900 \mathrm{~nm}$ for experiments involving GFP. The microscope was equipped with a primary dichroic mirror at $695 \mathrm{~nm}$ and green and red fluorescence was split and filtered using a secondary dichroic at $560 \mathrm{~nm}$ and two bandpass emission filters: $525-40 \mathrm{~nm}$ and 605-70 nm (Chroma Technologies).

Images analysis

FITC-injected animals: Images were analyzed using ImageJ. Vessel area was obtained from the FITC-labelled lumen. Each image was thresholded and a particle analysis was performed. No animals were excluded from the analysis.

AAV-injected mice: To automate the tracking blood-vessel cross-sectional areas and widths, we have opted for using an algorithm which combines the advantages of human blood vessel detection in the initial step, while automating the tracking across multiple frames through the use of an image registration algorithm (Extended data Figure 6). In the first step, human input is required to define a number of Regions of Interest (ROI's) from either an initial frame, or an average of multiple successive frames. These ROls correspond to blood vessel cross sections taken at different imaging angles and will form the gray-scale template matrix $T_{i j}^{R O I}$. After selecting the ROls, the user manually selects a boundary by selecting a user specified number of polygonal vertices that surround the to-be-tracked area. The interior of the polygon generated by these vertices forms an area mask, which is computed by the inpoylgon in MATLAB 2019 . The result is a binary matrix $M_{i j}^{R O I}$ for each ROI template, where $M_{i j}^{R O I}=1$ if pixel $(i, j)$ is within the designated area, and 0 otherwise.

With the template and mask matrices created for each ROI, frames for each recording are loaded successively creating a sub-matrix for each ROI for the $k^{\text {th }}$ frame, $C_{i j}^{R O I, k}$. Thirion's DEMONS algorithm is then used find the distortion function $D^{R O I, k}$ which transforms the $T^{R O I}$ into $C^{R O I, k}$ for each new frame $\mathrm{k}^{122,123}$, as implemented with the default options in MATLAB 2019. 
The distortion function, $D^{R O I, k}$ is subsequently used to distort the Mask $M^{R O I}$ (imregdemons), creating $\widehat{M}^{R O I, k}$ The cross-sectional area for each frame $k$ is then estimated with:

$A^{R O I, k}=\sum_{i, j} \widehat{M}_{i j}^{R O I, k}$

The intensity of the imaged area is computed as:

$I^{R O I, k}=\sum_{i, j} \widehat{M}_{i j}^{R O I, k} \cdot C_{i j}^{R O I, k}$

The cross-sectional width for cylindrical vessels is estimated by first extracting out the contour $\left(x_{i}, y_{i}\right), i=1,2, \ldots m$ from the surrounding mask for the $k^{t h}$ frame using the contourc function applied to $\widehat{M}^{R O I, k}$. Then, we construct a finite difference estimate of the local derivative using a second order differencing scheme centered around the point $\left(x_{j}, y_{j}\right)$ to estimate the local slope of the contour:

$m=\frac{x_{j+\Delta}-x_{j-\Delta}}{y_{j+\Delta}-y_{j-\Delta}}$

With the slope of the contour determined, we construct an orthogonal line to the contour using $y=y_{j}-\frac{x-x_{j}}{m}$, and use this tangent line to detect the second intersection point with the contour surface, $\left(x_{j^{\prime}}, y_{j^{\prime}}\right)$. The cross-sectional distance is then estimated to be $d=\sqrt{\left(y_{j}-y_{j^{\prime}}\right)^{2}+\left(x_{j}-x_{j^{\prime}}\right)^{2}}$

Two points are chosen as $\left(x_{j}, y_{j}\right)$, the point where the tangent distance is computed: The first is the point $\left(x_{j}, y_{j}\right)$ on the contour such that $x_{j}$ is nearest to $x_{m}=\bar{x}_{i}$ the average $\mathrm{x}$-coordinate on the contour, while the second point is the point $\left(x_{j}, y_{j}\right)$ such that $y_{j}$ is nearest to $y_{m}=\bar{y}_{J}$, the average $y$-coordinate on the contour. One of these two points can estimate the cross-sectional width of a cylindrical blood vessel dependent on its orientation. For the same vessel analyzed 5 times (mean $\pm S D$ ): $9.0 \pm 0.8$. Animals were excluded from the analysis if the signal from the vessel was too weak to allow proper tracking from the algorithm.

\section{Calculations}


For the responses to drug superfusion and whisker stimulation during awake two-photon imaging, the vessel area was first analyzed as described in the 'image analysis' section. The average area was calculated for the baseline ( $5 \mathrm{sec}$ for the whisker stimulation and 5 min for the drug superfusion). The area value after the baseline were subtracted from the baseline and a ratio was made on the baseline value and multiplied by 100 : Vessel size $(\%)=$ area baseline (area baseline - area) / area baseline $\times 100$. The maximum dilation represents the peak area for individual experiments after the baseline. For the drug application, each value represents one vessel. For the whisker stimulation, each value represents the average of the response to 3 stimulations for one vessel. The number of vessels and animals used are specified in the figure legend for each experiment.

Representative images for figures

Every image presented in the figures for the drugs application and the response to whisker stimulation were processed the same way. Two images were averaged and a Gaussian filter ( 0.5 or 1 pixel radius) was performed by ImageJ plugins.

\section{Pressure Myography}

Vessel Isolation

Male C57BL/6 mice between 6 and 8 weeks of age were injected intraperitoneally with sodium pentobarbital (50 mg/kg) to induce surgical anesthesia (i.e., stage 3, loss of blink reflex). A total of 12 animals were used to obtain the cerebral arteries (posterior and middle cerebral) described in this study. No animals were excluded from the analysis. To isolate cerebral arteries, anesthetized animals were first euthanized by decapitation, and the excised brain was placed in a cooled dissection chamber.

\section{Arterial Pressure Myography}

After isolation, arteries were cannulated on glass pipettes fitted in a pressure myography chamber (Living Systems, Burlington, VT). The vessel lumen was filled with Kreb's buffer (115 
$\mathrm{NaCl}, 5 \mathrm{mM} \mathrm{KCl}, 25 \mathrm{mM} \mathrm{NaHCO}_{3}, 1.2 \mathrm{mM} \mathrm{MgCl}_{2}, 2.5 \mathrm{mM} \mathrm{CaCl}_{2}, 1.2 \mathrm{mM} \mathrm{KH}_{2} \mathrm{PO}_{4}$, and $10 \mathrm{mM}$ D-glucose) containing $1 \%$ bovine serum albumin, $\mathrm{pH}$ was adjusted to $7.4^{81,124}$. The cannulated vessel/chamber apparatus was placed on the stage of an inverted microscope, and the vessel was superfused with Kreb's buffer at a constant flow of $6-7 \mathrm{ml} / \mathrm{min}$ using a peristaltic pump and suction line. Bath solution was maintained at $37^{\circ} \mathrm{C}$ and gassed with $95 \%$ air/5\% carbon dioxide. After 5-10 minutes of equilibration, the intraluminal pressure of cannulated vessels was increased in a stepwise manner and then maintained at $70 \mathrm{mmHg}$. The vessels typically developed myogenic tone within 20-30 minutes. Continuous video measurement of the intraluminal vessel diameter was carried out using a diameter tracking system (IonOptix, Milton, MA). Drug-containing solutions were added to the bath through the perfusion pump. For endothelial denudation, an air bubble was slowly passed through the lumen of the vessel after cannulation of one end of the vessel. Physiological saline was then gently passed through the lumen to flush out any cellular debris. The vessel was then fully cannulated and allowed to equilibrate as described above before the application of intraluminal pressure and the development of myogenic tone. Drug induced-changes in vessel internal diameter were calculated and are presented as the absolute change (in microns) from the baseline intraluminal diameter recorded at steady state myogenic tone immediately prior to drug application. Reagents

Bradykinin, EGTA (ethylene glycol-bis(2-aminoethylether)-N,N,N',N'-tetraacetic acid), Phenylephrine hydrochloride ((R)-(-)-1-(3-hydroxyphenyl)-2-methylaminoethanol hydrochloride), (-)Norepinephrine bitartrate salt, 4-AP (4-aminopyridine), SNP (sodium nitroprusside), DMSO (dimethyl sulfoxide), Pinacidil (N-cyano-N0-4pyridinyl-N"-(1,2,2-trimethylpropyl)guanidine monoh ydrate), and all required chemicals were purchased from SigmaAldrich (Oakville, ON, Canada). Euthanyl (sodium pentobarbital, $250 \mathrm{mg} / \mathrm{ml}$ ) was purchased from Bimeda-MTC Animal Health Inc, Cambridge, ON, Canada. SKA-31 (naphtho [1, 2-d] thiazole-2-ylamine) was synthesized as previously described ${ }^{80}$. Reelin and MOCK media were prepared as described previously. SKA- 
31 and pinacidil were prepared as $10 \mathrm{mmol} / \mathrm{L}$ stock solutions in DMSO and then diluted directly into the external bath solution. The final concentration of DMSO reaching the tissue was typically $0.02 \%$ (vol/vol) or less. In preliminary experiments, we observed that a considerably higher concentration of DMSO $(0.2 \% \mathrm{v} / \mathrm{v}$ final $)$ had no effect on either basal myogenic tone or the responsiveness of cerebral arteries to vasodilator agents ${ }^{81}$.

\section{Cell cultures}

Mouse primary BECs (mBECs) were prepared using an adapted protocol ${ }^{125}$. No samples were excluded from the analysis. The brains of five C57BL6 mice aged between 2 and 6 months were used to cover a $75 \mathrm{~cm}^{2}$ flask. Mice were sacrificed under anesthesia with 2-bromo-2-chloro1,1,1-trifluoro-ethane (Sigma) by cervical dislocation. The brains were placed in petri dish filled with ice-cold PBS where the cerebellum was removed and discarded. Meninges were removed by rolling brains on UV sterilized $30 \times 30 \mathrm{~cm}$ kimwipes until they were no longer visible. The brains were transferred to a dry petri dish and diced thoroughly with a scalpel forming pieces about $1 \mathrm{~mm}^{3}$ in size. The homogenate was then added to the enzymatic digestion buffer (Worthington Biochemical Corp., $250 \mathrm{U} / \mathrm{ml}$ collagenase type II (LS004174), $10 \mathrm{U} / \mathrm{ml}$ dispase (LS02100) and $10 \mathrm{U} / \mathrm{ml}$ DNAase (LS006343) in DMEM) and incubated with agitation for $90 \mathrm{~min}$ at $37^{\circ} \mathrm{C}$. After the digestion, the homogenate was pelleted by centrifugation at $200 \mathrm{~g}$ for $3 \mathrm{~min}$., the supernatant was aspirated and the pellet was resuspended in $4^{\circ} \mathrm{C}$ DMEM and pelleted again at $200 \mathrm{~g}$ for $3 \mathrm{~min}$. The supernatant was aspirated and brain material was homogenized in DMEM $20 \%$ BSA. The suspension was then centrifuged at $1500 \mathrm{~g}$ for 20 min at $4^{\circ} \mathrm{C}$ pelleting the brain capillaries. The supernatant and floating white matter were aspirated and capillaries were resuspended in Medium 131 + MVGS supplement kit (Gibco, ThermoFisher, M-131-500 and S00525) and passed through a $70 \mu \mathrm{m}$ cell strainer to dissociate the capillary cells. The capillary cell suspension was then supplemented with $4 \mu \mathrm{g} / \mathrm{ml}$ of puromycin (Sigma Aldrich, P883310MG) plated on collagen IV (Santa Cruz Biotechnology, sc-29010) coated culture plates and 
placed in a $\mathrm{CO}_{2}$ incubator at $37^{\circ} \mathrm{C}$. After 2 days the media was changed to puromycin-free growth media and cells were cultured until reaching appropriate confluency.

hBECs (hCMEC/D3, a generous gift from Dr Amir Nezhad, University of Calgary) were cultured according to the supplier protocol (EMD Millipore, SCC066), in EndoGRO ${ }^{\mathrm{TM}}$-MV Complete Media Kit (EMD Millipore, SCME004). Cells were plated on Collagen I (EMD Millipore, 08-115) coated T75 culture flasks and passaged at $90 \%$ confluency.

Transfection of siRNA and DNA vectors

hBECs and mBECs were transfected with human CD2AP siRNA (Santa Cruz Biotechnology, sc29984) and mouse CD2AP siRNA (Santa Cruz Biotechnology, sc-29985), respectively. Both siRNA efficiently reduced the levels of CD2AP and its target, the ApoER2. A third efficient siRNA (ON-TARGETplus, Dharmacon, L-041647-01-0005) confirmed the knockdown of CD2AP in mBECs. For the mBECs, the transfection was performed 5 days after plating the cells, yielding a confluency of about $60 \%$, using HiPerFect (QIAGEN) reagent according to the manufacturer instructions. For one well of a 24 -well plate, $10 \mathrm{nM}$ siRNA were added to the well. For both cell models, transfection media was aspirated and replaced with growth media after 3 hours. Cells were harvested, fixed or used for assays 42 hours and 7 days post-transfection for hBECs and mBECs, respectively.

Reelin treatment of $h B E C s$

hBECs cells were first transfected with control or CD2AP siRNA according to the method describe above. After the transfection, hBECs cells were incubated for 60 min in DMEM media without FBS. The media was then replaced with DMEM with Reelin (20 nM) or control solution for 30 or $120 \mathrm{~min}$. 


\section{Immunostaining for cell culture}

Immunochemistry was performed based on published protocol ${ }^{72,117}$. BECs were rinsed in HBSS and fixed in 4\% PFA for $15 \mathrm{~min}$ at room temperature. The immunostaining was performed using to the same protocol as the brain slices. The cells were mounted and visualized using a Nikon D-Eclipse C1si Spectral Laser confocal microscope. The list of primary antibodies is available in Extended data Table 3.

\section{Cell culture and mouse tissue western blotting}

Cells were lysed directly on plate in RIPA Buffer (50 mM Tris-Cl ph 8.0, $150 \mathrm{mM} \mathrm{NaCl}, 1 \%$ Triton X-100, 0.5\% sodium deoxycholate, $0.1 \%$ SDS, 1 mM EDTA, 0.2 mM PMSF, cOmplete ${ }^{\text {TM }}$ tablet $^{2}$ (Roche)) using a cell scrapper. Mouse brain samples were homogenized using a Dounce tissue grinder with ice-cold RIPA buffer. For both, cells and mouse brain samples, homogenates were sonicated and centrifuged for $15 \mathrm{~min}$ at 13,000 rpm. Protein concentration was determined by DC assay (BioRad Laboratories) according to the manufacturer instructions. Proteins were separated using SDS-PAGE and transferred to PVDF membranes (Millipore) for Western blot analysis. Membranes were blocked using 5\% skim-milk powder and incubated overnight in primary antibodies. Secondary anti-HRP conjugate antibodies were used and immunoreactivity was visualized using Western LightingTM (PerkinElmer). Immunoreactivity was developed on film or using a ChemiDoc ${ }^{\mathrm{TM}}$ imaging system (Biorad), band intensity was quantified using Image Lab and normalized to actin as a loading control. The list of primary antibodies is available in Extended data Table 3.

\section{Immunoprecipitations (IP)}

From cell lysates

Proteins were extracted from cells as described in the Western blotting section. $1 \mathrm{mg}$ of protein in $500 \mu$ of homogenization buffer (wash buffer with different concentration of ionic and non- 
ionic detergents, see Figure 2 for detergent content) was added to $10 \mu \mathrm{l}$ of packed beads: Protein G Sepharose 4 Fast Flow (GE Healthcare Life Sciences) for mouse antibodies or Protein A Sepharose 4 Fast Flow (GE Healthcare Life Sciences) for rabbit antibodies. Sample were inverted at $4^{\circ} \mathrm{C}$ for 1 hour as a preclear step. At the same time $1 \mu \mathrm{g}$ of antibody in $500 \mu \mathrm{l}$ of IP wash buffer (20 mM Tris-Cl ph 7.5, $150 \mathrm{mM} \mathrm{NaCl,} \mathrm{0.1 \%} \mathrm{Triton} \mathrm{X-100,} \mathrm{0.1 \%} \mathrm{NP40,} 0.5 \mathrm{mM}$ EDTA, $0.2 \mathrm{mM}$ PMSF, cOmplete ${ }^{\mathrm{TM}}$ tablet (Roche)) was added to $10 \mu \mathrm{l}$ of packed beads and inverted at $4^{\circ} \mathrm{C}$ for 1 hour to bind the antibodies to the beads. The tubes were centrifuged to pellet the beads and the antibody solutions were aspirated and replaced with precleared cell lysate and inverted at $4^{\circ} \mathrm{C}$ for 2 hours to immunoprecipitate the protein of interest. Beads were rinsed 3 times with IP wash buffer and $40 \mu$ of $2 x$ Laemmli Buffer was added to packed beads and boiled for $5 \mathrm{~min}$. at $95^{\circ} \mathrm{C}$ pelleted and supernatant used for Western blot.

From mouse brain homogenates

All steps are identical to the cell lysate IP except that whole mouse brain is homogenized in a Dounce tissue grinder on ice. The homogenate was fractionated into two fractions ${ }^{126}$. The first fraction was generated by homogenizing in brain fraction 1 (BF1) buffer (150 mM NaCl, $1 \%$ NP40, $50 \mathrm{mM}$ Tris- $\mathrm{Cl}(\mathrm{pH} 8.0)$, cOmplete ${ }^{\mathrm{TM}}$ tablet (Roche)) and centrifuged for $15 \mathrm{~min}$. at 12,000g. The supernatant was designated as BF1 and the pellet was resuspended in BF2 buffer (150 mM

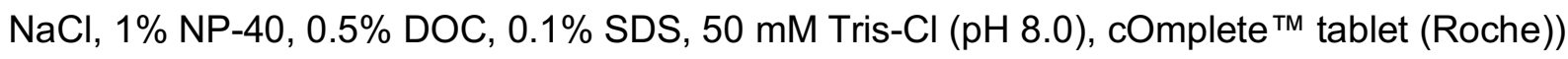
and centrifuged at $10,000 \mathrm{~g}$ and the supernatant designated as BF2.

\section{AAV preparation}

\section{Plasmid construction}

The DNA sequences encoding the mouse U6 promoter, CMV-eGFP and WPRE from pLL3.7 (a gift from Luk Parijs (Addgene plasmid \# 11795 ; http://n2t.net/addgene:11795 ;

RRID:Addgene_11795) ${ }^{127}$ were subcloned into the Mlul and Xhol restriction sites of 
pENN.AAV.hSyn.Cre.WPRE.hGH (a gift from James M. Wilson (Addgene plasmid \# 105553 ; http://n2t.net/addgene:105553 ; RRID:Addgene_105553)), replacing the hSyn.cre.WPRE with U6.CMV.eGFP.WPRE to generate pAAV.U6.CMV.GFP.WPRE.hGH. The siRNA sequences purchased from Santa Cruz Biotechnology and Dharmacon that had previously been validated in vitro to efficiently knockdown CD2AP, were engineered into shRNA 97-mer backbones ${ }^{128}$,, synthesized into a large primer and inserted immediately downstream of the U6 promoter to generate pAAV.U6.shRNA.CMV.GFP.WPRE.hGH using the NEBuilder hifi DNA assembly cloning kit (New England Biolabs). The sequences of all constructs were verified by Sanger DNA sequencing.

\section{AAV production}

$A A V$ viral vectors containing the AAV-BR1 capsid were generated using the methods of Challis et. al. ${ }^{129}$. Briefly, 293FT cells (Thermofisher) were grown to $\sim 90 \%$ confluency in Corning hyperflasks (Corning) and co-transfected with $129 \mu \mathrm{g}$ pHELPER (Agilent), $238 \mu \mathrm{g}$ rep-cap plasmid encoding AAV-BR ${ }^{48}$ and $64.6 \mu \mathrm{g}$ equimolar mixtures of pAAV.U6.shRNA.CMV.GFP.WPRE.hGH corresponding to either the four Dharmacon shRNA sequences, the three Santa Cruz shRNA sequences or a scrambled control using the PElpro transfection reagent (Polyplus). AAVs were precipitated from media harvested after 3 days and 5 days using $40 \% \mathrm{PEG} / 2.5 \mathrm{M} \mathrm{NaCl}$ and pooled the cells harvested after 5 days in buffer containing $500 \mathrm{mM} \mathrm{NaCl}, 40 \mathrm{mM}$ Tris Base and $10 \mathrm{mM} \mathrm{MgCl}_{2}$. The lysate was incubated with $100 \mathrm{U} / \mathrm{ml}$ salt-active nuclease (Arcticzymes) at $37^{\circ} \mathrm{C}$ for $1 \mathrm{~h}$ and then centrifuged at $2000 \mathrm{~g}$ for 15 min. AAV was purified from the resulting lysate using an iodixanol step gradient containing $15,25,40$ and $60 \%$ iodixanol ${ }^{129}$ in optiseal tubes (Beckman) followed by centrifugation at 350,000 g using a Type $70 \mathrm{Ti}$ ultracentrifuge rotor (Beckman). Following centrifugation, the AAVs were harvested from the $40 \%$ layer using a $10 \mathrm{cc}$ syringe and 16 -gauge needle, diluted in PBS containing $0.001 \%$ pluronic F68 (Gibco) and filtered using a $0.2 \mu \mathrm{m}$ syringe filter. The AAVs were concentrated and buffer-exchanged by 5 rounds of centrifugation using Amicon Ultra-15 
$100 \mathrm{kDa}$ molecular weight cut off centrifugal filter units (Millipore). The titer was determined using the qPCR Adeno-Associated Virus Titration kit (Applied Biological Materials) and the purity was verified by SDS-PAGE and total protein staining using instant blue reagent (Expedeon). AAV was administered to 30 days old male C57BL6 mice with a retro-orbital injection (150 $\mu$, $\left.1 \times 10^{11} \mathrm{GC}\right)$.

\section{MRI}

14 days and 21 days after AAV injection, mice were subjected to MRI according to a previously published protocol ${ }^{130}$. One mouse was excluded from the analysis because of a tumor. Briefly, mice were spontaneously ventilated with a mixture of $2 \%$ isoflurane, $70 \% \mathrm{~N}_{2}$ and $30 \% \mathrm{O}_{2}$. The mouse was set up in the MRI system on top of a heating pad to maintain body temperature at $37^{\circ} \mathrm{C}$. A thermometer with a rectal probe allowed to follow body temperature. Breathing rate was monitored continuously. Imaging was performed using a 9.4T, $210 \mathrm{~mm}$ bore Magnex magnet, run by Bruker Avance II hardware controlled by Bruker ParaVision 5.1 software (Bruker Avance console, Bruker BiospinGmbH, Rheinstetten, Germany).

Perfusion was measured by acquiring axial slices around bregma $-1.94 \mathrm{~mm}$ using a CASLHASTE sequence: $\mathrm{TR}=3000 \mathrm{~ms}, \mathrm{TE}=13.5 \mathrm{~ms}, \mathrm{FOV}=25.6 \times 25.6 \mathrm{~mm}$, matrix size $=128 \times 128$ pixels, slice thickness $=1 \mathrm{~mm}, 16$ averages. Two control and two tagged images were collected per measurement. Following these, a T1 map was obtained using a RARE-VTR sequence:

$\mathrm{TE}=10 \mathrm{~ms}, \mathrm{TR}=100,500,1000,3000$, and $7500 \mathrm{~ms}$.

Quantification of the perfusion images and T1 map were done using a custom Matlab script ${ }^{131}$,

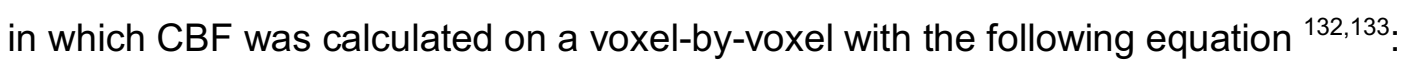

$\mathrm{f}=\frac{1}{T_{1}} \frac{\lambda}{2 \alpha} y_{j}-\left(\frac{M_{c}-M_{l}}{M_{c}}\right)$

Were $\mathrm{f}$ is the tissue perfusion ( $\mathrm{ml} / 100 \mathrm{~g} / \mathrm{min}$.), $\lambda(0.9)$ is the blood-brain partition coefficient ${ }^{134,135}$, $M_{c}$ is the average value for the control images, $M_{l}$ is the average value for the tagged images, $\alpha$ 
$(0.675 \pm 0.044)$ is the efficiency of the spin labelling ${ }^{131}$ and $T_{1}$ is the $T_{1}$ value for each voxel. A ROI was generated using a tagged image and was then used on the $T_{1}$ map to obtain a mean \pm SD for perfusion. Each ROI was traced 3 times and the values presented in the figure is the average of these $3 \mathrm{ROI}$.

\section{Human brain samples}

The brain parietal cortices samples used for this study were provided from the cohort of volunteers from the Religious Order Study, a longitudinal clinical-pathologic study of aging and dementia. An extensive amount of clinical and neuropathological data published from that cohort are available ${ }^{40,136,137}$. Volunteers characteristics are available in Table S1 and were published previously ${ }^{40,138}$. Participants to this study were recruited without known dementia and underwent a clinical evaluation every year until death. Cognitive function of participants was evaluated using 21 cognitive performance test that were evaluated by a clinical neuropsychologist and expert clinician ${ }^{39}$. AD diagnosis was assessed based on a significant decline in at least two cognitive domains including episodic memory. Participants were classified in the $\mathrm{MCl}$ group if they showed cognitive impairment but were not diagnosed with dementia ${ }^{139}$ and $\mathrm{NCl}$ volunteers had no cognitive impairment ${ }^{140}$. Using 19 cognitive tests, a global cognitive score and a score for five different cognitive domains (episodic, semantic and working memory, perceptual speed and visual-spatial ability) was generated ${ }^{141}$. The volunteers were interviewed about use of antihypertensive and diabetes medication during the two weeks before each evaluation ${ }^{142,143}$. The neuropathological diagnosis was based on a blinded evaluation of the $A B C$ scoring method from the National Institute of Aging - Alzheimer's Association (NIA-AA) guideline ${ }^{144}$ : A) Thal score (accumulation of $A \beta$ plaques) ${ }^{145}$, B) Braak score (neurofibrillary tangle pathology) ${ }^{146}$, and C) CERAD score (neuritic plaques pathology) ${ }^{147}$. Participants from this cohort were also characterized for the presence of cortical infarcts and CAA ${ }^{15}$. 
Preparation of whole homogenates and vessel-enriched extracts from human parietal cortex

Cortical extracts were prepared as described previously ${ }^{138}$. Brain vessel-enriched extracts from frozen human parietal cortex samples were prepared according to Bourassa et al., ${ }^{15,41}$. Briefly, parietal cortex samples were thawed on ice in a vessel isolation buffer (MIB; 15 mM HEPES, $147 \mathrm{mM} \mathrm{NaCl}, 4 \mathrm{mM} \mathrm{KCl}, 3 \mathrm{mM} \mathrm{CaCl}$ and $12 \mathrm{mM} \mathrm{MgCl}_{2}$ ) containing protease and phosphatase inhibitor cocktails (Bimake, Houston, TX), meninges and white matter were removed, and were then homogenized in MIB and spun at $1,000 \mathrm{~g}$ for 10 minutes at $4^{\circ} \mathrm{C}$. The pellet was then homogenized in MIB containing 18\% dextran (from leuconostoc mesenteroides, M.W. 60,000 90,000; Sigma-Aldrich) and centrifuged at $4,000 \mathrm{~g}$ for $20 \mathrm{~min}$. at $4^{\circ} \mathrm{C}$. The pellet was resuspended in MIB and filtered through a $20 \mu \mathrm{m}$ nylon filter (Millipore). Vessels, retained on the filter, were homogenized in lysis buffer $\left(150 \mathrm{mM} \mathrm{NaCl}, 10 \mathrm{mM} \mathrm{NaH}_{2} \mathrm{PO}_{4}, 1 \%\right.$ Triton X-100, 0.5\% SDS and $0.5 \%$ sodium deoxycholate), sonicated and spun at $100,000 \mathrm{~g}$ for $20 \mathrm{~min}$. at $4^{\circ} \mathrm{C}$. The resulting supernatant was concentrated using a Vivaspin device (MWCO, 3 kDa; Sartorius Stedim Biotech) and kept for Western immunoblotting analyses. In addition, the eluate containing the post-vascular fraction was pelleted following centrifugation at $16,000 \mathrm{~g}$ for $20 \mathrm{~min}$. at $4^{\circ} \mathrm{C}$, homogenized in lysis buffer and spun again at $100,000 \mathrm{~g}$ for $20 \mathrm{~min}$. at $4^{\circ} \mathrm{C}$. The resulting supernatant was preserved for Western immunoblotting analyses. Protein concentrations in all fractions were measured using the bicinchoninic acid assay (Thermo Fisher Scientific). $1 \mathrm{NCl}, 2 \mathrm{MCl}$ and $1 \mathrm{AD}$ samples were lost during the sample preparation.

\section{Statistical Analysis}

Data are presented as mean \pm SEM. Details for the statistical analysis for each experiment are described in the figure legends. For all data, when two groups were compared, a Student t-test (paired or unpaired when appropriate) was performed. A one-way ANOVA with Tukey's post hoc analysis was utilized when comparing multiple groups. When the variance was not equal, a Welch's correction was applied. For the cell experiments, protein levels after the CD2AP 
knockdown were compared to a theoretical mean of 100 using a one-sample t-test. For the CD2AP-depleted cells treated with Reelin or control experiments, the response after 30 and 120 min. were compared to the zero value using a one-sample t-test and the cells transfected with the CD2AP siRNA were compared to the cells transfected with control siRNA using an unpaired Student-test. To verify the association between the two parameters, a linear regression was used to generate a correlation coefficient. Values of $\mathrm{P}<0.05$ were accepted as statistically significant. All statistical analyses were performed with Prism 7 (GraphPad software) and SPSS (IBM) softwares. 


\section{Bibliography}

1. Association, A. 2020 Alzheimer's Disease Facts and Figures. Alzheimers Dement 16, 391 (2020).

2. Kisler, K., Nelson, A. R., Montagne, A. \& Zlokovic, B. V. Cerebral blood flow regulation and neurovascular dysfunction in Alzheimer disease. Nat Rev Neurosci 18, 419-434 (2017).

3. Korte, N., Nortley, R. \& Attwell, D. Cerebral blood flow decrease as an early pathological mechanism in Alzheimer's disease. Acta Neuropathol (2020).

4. Chételat, G. et al. Mild cognitive impairment: Can FDG-PET predict who is to rapidly convert to Alzheimer's disease. Neurology 60, 1374-1377 (2003).

5. Sweeney, M. D., Kisler, K., Montagne, A., Toga, A. W. \& Zlokovic, B. V. The role of brain vasculature in neurodegenerative disorders. Nat Neurosci 21, 1318-1331 (2018).

6. Montagne, A. et al. Blood-brain barrier breakdown in the aging human hippocampus. Neuron 85, 296-302 (2015).

7. Iturria-Medina, Y. et al. Early role of vascular dysregulation on late-onset Alzheimer's disease based on multifactorial data-driven analysis. Nat Commun 7, 11934 (2016).

8. Nation, D. A. et al. Blood-brain barrier breakdown is an early biomarker of human cognitive dysfunction. Nat Med 25, 270-276 (2019).

9. Leeuwis, A. E. et al. Lower cerebral blood flow is associated with impairment in multiple cognitive domains in Alzheimer's disease. Alzheimers Dement 13, 531-540 (2017).

10. Love, S. \& Miners, J. S. Cerebrovascular disease in ageing and Alzheimer's disease. Acta Neuropathol 131, 645-658 (2016).

11. Montagne, A., Zhao, Z. \& Zlokovic, B. V. Alzheimer's disease: A matter of blood-brain barrier dysfunction. J Exp Med 214, 3151-3169 (2017).

12. Montagne, A. et al. APOE4 leads to blood-brain barrier dysfunction predicting cognitive decline. Nature 581, 71-76 (2020).

13. Montaldi, D. et al. Measurements of regional cerebral blood flow and cognitive performance in Alzheimer's disease. J Neurol Neurosurg Psychiatry 53, 33-38 (1990).

14. Sweeney, M. D., Zhao, Z., Montagne, A., Nelson, A. R. \& Zlokovic, B. V. Blood-Brain Barrier: From Physiology to Disease and Back. Physiol Rev 99, 21-78 (2019).

15. Bourassa, P., Tremblay, C., Schneider, J. A., Bennett, D. A. \& Calon, F. Beta-amyloid pathology in human brain microvessel extracts from the parietal cortex: relation with cerebral amyloid angiopathy and Alzheimer's disease. Acta Neuropathol 137, 801-823 (2019).

16. Storck, S. E. et al. Endothelial LRP1 transports amyloid- $\beta(1-42)$ across the blood-brain barrier. J Clin Invest 126, 123-136 (2016).

17. Winkler, E. A. et al. GLUT1 reductions exacerbate Alzheimer's disease vasculo-neuronal dysfunction and degeneration. Nat Neurosci 18, 521-530 (2015).

18. Kobayashi, S., Sawano, A., Nojima, Y., Shibuya, M. \& Maru, Y. The c-Cbl/CD2AP complex regulates VEGF-induced endocytosis and degradation of FIt-1 (VEGFR-1). FASEB J 18, 929-931 (2004).

19. Lynch, D. K. et al. A Cortactin-CD2-associated protein (CD2AP) complex provides a novel link between epidermal growth factor receptor endocytosis and the actin cytoskeleton. $J$ Biol Chem 278, 21805-21813 (2003).

20. Bertram, L., McQueen, M. B., Mullin, K., Blacker, D. \& Tanzi, R. E. Systematic metaanalyses of Alzheimer disease genetic association studies: the AlzGene database. Nat Genet 39, 17-23 (2007).

21. Chen, H. et al. Analyzing 54,936 Samples Supports the Association Between CD2AP rs9349407 Polymorphism and Alzheimer's Disease Susceptibility. Mol Neurobiol 52, 1-7 (2015). 
22. Hollingworth, $P$. et al. Common variants at ABCA7, MS4A6A/MS4A4E, EPHA1, CD33 and CD2AP are associated with Alzheimer's disease. Nat Genet 43, 429-435 (2011).

23. Lambert, J. C. et al. Meta-analysis of 74,046 individuals identifies 11 new susceptibility loci for Alzheimer's disease. Nat Genet 45, 1452-1458 (2013).

24. Naj, A. C. et al. Effects of multiple genetic loci on age at onset in late-onset Alzheimer disease: a genome-wide association study. JAMA Neurol 71, 1394-1404 (2014).

25. Vardarajan, B. N. et al. Rare coding mutations identified by sequencing of Alzheimer disease genome-wide association studies loci. Ann Neurol 78, 487-498 (2015).

26. Cormont, M. et al. CD2AP/CMS regulates endosome morphology and traffic to the degradative pathway through its interaction with Rab4 and c-Cbl. Traffic 4, 97-112 (2003).

27. Rajendran, L. et al. Efficient inhibition of the Alzheimer's disease beta-secretase by membrane targeting. Science 320, 520-523 (2008).

28. Furusawa, K. et al. CD2-associated protein (CD2AP) overexpression accelerates amyloid precursor protein (APP) transfer from early endosomes to the lysosomal degradation pathway. J Biol Chem 294, 10886-10899 (2019).

29. Liao, F. et al. Effects of CD2-associated protein deficiency on amyloid- $\beta$ in neuroblastoma cells and in an APP transgenic mouse model. Mol Neurodegener 10, 12 (2015).

30. Ubelmann, F. et al. Bin1 and CD2AP polarise the endocytic generation of beta-amyloid. EMBO Rep 18, 102-122 (2017).

31. Shulman, J. M. et al. Genetic susceptibility for Alzheimer disease neuritic plaque pathology. JAMA Neurol 70, 1150-1157 (2013).

32. Tsuji, K. et al. Re-characterization of the Glomerulopathy in CD2AP Deficient Mice by High-Resolution Helium Ion Scanning Microscopy. Sci Rep 7, 8321 (2017).

33. Olin, J. W. et al. A plasma proteogenomic signature for fibromuscular dysplasia. Cardiovasc Res 116, 63-77 (2020).

34. Stage, E. et al. The effect of the top 20 Alzheimer disease risk genes on gray-matter density and FDG PET brain metabolism. Alzheimers Dement (Amst) 5, 53-66 (2016).

35. Yao, $X$. et al. Targeted genetic analysis of cerebral blood flow imaging phenotypes implicates the INPP5D gene. Neurobiol Aging 81, 213-221 (2019).

36. Lehtonen, $\mathrm{S}$. et al. CD2-associated protein is widely expressed and differentially regulated during embryonic development. Differentiation 76, 506-517 (2008).

37. Li, C., Ruotsalainen, V., Tryggvason, K., Shaw, A. S. \& Miner, J. H. CD2AP is expressed with nephrin in developing podocytes and is found widely in mature kidney and elsewhere. Am J Physiol Renal Physiol 279, F785-92 (2000).

38. Vanlandewijck, M. et al. A molecular atlas of cell types and zonation in the brain vasculature. Nature 554, 475-480 (2018).

39. Bennett, D. A. et al. Decision rules guiding the clinical diagnosis of Alzheimer's disease in two community-based cohort studies compared to standard practice in a clinic-based cohort study. Neuroepidemiology 27, 169-176 (2006).

40. Tremblay, C. et al. Association of Neuropathological Markers in the Parietal Cortex With Antemortem Cognitive Function in Persons With Mild Cognitive Impairment and Alzheimer Disease. J Neuropathol Exp Neurol (2017).

41. Bourassa, P., Tremblay, C., Schneider, J. A., Bennett, D. A. \& Calon, F. Brain mural cell loss in the parietal cortex in Alzheimer's disease correlates with cognitive decline and TDP. 43 pathology. Neuropathol Appl Neurobiol (2020).

42. Shulman, J. M. et al. Functional screening in Drosophila identifies Alzheimer's disease susceptibility genes and implicates Tau-mediated mechanisms. Hum Mol Genet 23, 870877 (2014).

43. Zhang, Y. et al. An RNA-sequencing transcriptome and splicing database of glia, neurons, and vascular cells of the cerebral cortex. J Neurosci 34, 11929-11947 (2014). 
44. Shih, N. Y. et al. Congenital nephrotic syndrome in mice lacking CD2-associated protein. Science 286, 312-315 (1999).

45. Grunkemeyer, J. A., Kwoh, C., Huber, T. B. \& Shaw, A. S. CD2-associated protein (CD2AP) expression in podocytes rescues lethality of CD2AP deficiency. J Biol Chem 280, 29677-29681 (2005).

46. Cochran, J. N., Rush, T., Buckingham, S. C. \& Roberson, E. D. The Alzheimer's disease risk factor CD2AP maintains blood-brain barrier integrity. Hum Mol Genet 24, 6667-6674 (2015).

47. Dustin, M. L. et al. A novel adaptor protein orchestrates receptor patterning and cytoskeletal polarity in T-cell contacts. Cell 94, 667-677 (1998).

48. Körbelin, J. et al. A brain microvasculature endothelial cell-specific viral vector with the potential to treat neurovascular and neurological diseases. EMBO Mol Med 8, 609-625 (2016).

49. ladecola, C. The Neurovascular Unit Coming of Age: A Journey through Neurovascular Coupling in Health and Disease. Neuron 96, 17-42 (2017).

50. Janik, R. et al. Attenuation of functional hyperemia to visual stimulation in mild Alzheimer's disease and its sensitivity to cholinesterase inhibition. Biochim Biophys Acta 1862, 957965 (2016).

51. Montagne, A. et al. Brain imaging of neurovascular dysfunction in Alzheimer's disease. Acta Neuropathol 131, 687-707 (2016).

52. Boylan, M. A. et al. Greater BOLD Variability is Associated With Poorer Cognitive Function in an Adult Lifespan Sample. Cereb Cortex (2020).

53. Tran, C. H. T., Peringod, G. \& Gordon, G. R. Astrocytes Integrate Behavioral State and Vascular Signals during Functional Hyperemia. Neuron 100, 1133-1148.e3 (2018).

54. Yamazaki, Y., Zhao, N., Caulfield, T. R., Liu, C. C. \& Bu, G. Apolipoprotein E and Alzheimer disease: pathobiology and targeting strategies. Nat Rev Neurol 15, 501-518 (2019).

55. Monsell, S. E. et al. Genetic Comparison of Symptomatic and Asymptomatic Persons With Alzheimer Disease Neuropathology. Alzheimer Dis Assoc Disord 31, 232-238 (2017).

56. Lazaris, A. et al. Alzheimer risk genes modulate the relationship between plasma apoE and cortical PiB binding. Neurol Genet 1, e22 (2015).

57. Chen, Y., Durakoglugil, M. S., Xian, X. \& Herz, J. ApoE4 reduces glutamate receptor function and synaptic plasticity by selectively impairing ApoE receptor recycling. Proc Natl Acad Sci U S A 107, 12011-12016 (2010).

58. Fuchigami, T. et al. Dab1-mediated colocalization of multi-adaptor protein CIN85 with Reelin receptors, ApoER2 and VLDLR, in neurons. Genes Cells 18, 410-424 (2013).

59. Yuan, W. \& Song, C. The Emerging Role of Rab5 in Membrane Receptor Trafficking and Signaling Pathways. Biochem Res Int 2020, 4186308 (2020).

60. Hoe, H. S. \& Rebeck, G. W. Regulation of ApoE receptor proteolysis by ligand binding. Brain Res Mol Brain Res 137, 31-39 (2005).

61. Lane-Donovan, C. \& Herz, J. The ApoE receptors VIdlr and Apoer2 in central nervous system function and disease. J Lipid Res 58, 1036-1043 (2017).

62. Mata-Balaguer, T., Cuchillo-Ibañez, I., Calero, M., Ferrer, I. \& Sáez-Valero, J. Decreased generation of $C$-terminal fragments of ApoER2 and increased reelin expression in Alzheimer's disease. FASEB J fj201700736RR (2018).

63. Lane-Donovan, C. et al. Reelin protects against amyloid $\beta$ toxicity in vivo. Sci Signal 8 , ra67 (2015).

64. Pujadas, L. et al. Reelin delays amyloid-beta fibril formation and rescues cognitive deficits in a model of Alzheimer's disease. Nat Commun 5, 3443 (2014).

65. Beffert, $U$. et al. Reelin-mediated signaling locally regulates protein kinase B/Akt and glycogen synthase kinase 3beta. J Biol Chem 277, 49958-49964 (2002). 
66. Rouka, E. et al. Differential Recognition Preferences of the Three Src Homology 3 (SH3) Domains from the Adaptor CD2-associated Protein (CD2AP) and Direct Association with Ras and Rab Interactor 3 (RIN3). J Biol Chem 290, 25275-25292 (2015).

67. Cuitino, L. et al. ApoER2 is endocytosed by a clathrin-mediated process involving the adaptor protein Dab2 independent of its Rafts' association. Traffic 6, 820-838 (2005).

68. Lee, J. et al. Adaptor protein sorting nexin 17 regulates amyloid precursor protein trafficking and processing in the early endosomes. J Biol Chem 283, 11501-11508 (2008).

69. Sacharidou, A. et al. Antiphospholipid antibodies induce thrombosis by PP2A activation via apoER2-Dab2-SHC1 complex formation in endothelium. Blood 131, 2097-2110 (2018).

70. Santana, J. \& Marzolo, M. P. The functions of Reelin in membrane trafficking and cytoskeletal dynamics: implications for neuronal migration, polarization and differentiation. Biochem J 474, 3137-3165 (2017).

71. Beffert, U. et al. Modulation of synaptic plasticity and memory by Reelin involves differential splicing of the lipoprotein receptor Apoer2. Neuron 47, 567-579 (2005).

72. Kiroski, I. et al. Reelin Improves Cognition and Extends the Lifespan of Mutant Ndel1 Mice with Postnatal CA1 Hippocampus Deterioration. Cereb Cortex (2020).

73. Qiu, S. et al. Cognitive disruption and altered hippocampus synaptic function in Reelin haploinsufficient mice. Neurobiol Learn Mem 85, 228-242 (2006).

74. Quu, S., Zhao, L. F., Korwek, K. M. \& Weeber, E. J. Differential reelin-induced enhancement of NMDA and AMPA receptor activity in the adult hippocampus. $J$ Neurosci 26, 12943-12955 (2006).

75. Rogers, J. T. et al. Reelin supplementation enhances cognitive ability, synaptic plasticity, and dendritic spine density. Learn Mem 18, 558-564 (2011).

76. Segarra, M. et al. Endothelial Dab1 signaling orchestrates neuro-glia-vessel communication in the central nervous system. Science 361, (2018).

77. Smalheiser, N. R. et al. Expression of reelin in adult mammalian blood, liver, pituitary pars intermedia, and adrenal chromaffin cells. Proc Natl Acad Sci U S A 97, 1281-1286 (2000).

78. Sturm, L., van Elst, L. T., Fiebich, B., Wolkewitz, M. \& Hornig, T. Intra-day variations of blood reelin levels in healthy individuals. Arch Med Sci 16, 118-123 (2020).

79. Perez-Costas, E., Fenton, E. Y. \& Caruncho, H. J. Reelin expression in brain endothelial cells: an electron microscopy study. BMC Neurosci 16, 16 (2015).

80. Sankaranarayanan, A. et al. Naphtho[1,2-d]thiazol-2-ylamine (SKA-31), a new activator of $\mathrm{KCa} 2$ and $\mathrm{KCa} 3.1$ potassium channels, potentiates the endothelium-derived hyperpolarizing factor response and lowers blood pressure. Mol Pharmacol 75, 281-295 (2009).

81. Mishra, R. C., Wulff, H., Hill, M. A. \& Braun, A. P. Inhibition of Myogenic Tone in Rat Cremaster and Cerebral Arteries by SKA-31, an Activator of Endothelial KCa2.3 and KCa3.1 Channels. J Cardiovasc Pharmacol 66, 118-127 (2015).

82. Chow, B. W. et al. Caveolae in CNS arterioles mediate neurovascular coupling. Nature 579, 106-110 (2020).

83. Toth, P. et al. Purinergic glio-endothelial coupling during neuronal activity: role of P2Y1 receptors and eNOS in functional hyperemia in the mouse somatosensory cortex. $\mathrm{Am} J$ Physiol Heart Circ Physiol 309, H1837-45 (2015).

84. Ulrich, V. et al. Genetic variants of ApoE and ApoER2 differentially modulate endothelial function. Proc Natl Acad Sci U S A 111, 13493-13498 (2014).

85. Jiang, M. H., Kaku, T., Hada, J. \& Hayashi, Y. Different effects of eNOS and nNOS inhibition on transient forebrain ischemia. Brain Res 946, 139-147 (2002).

86. Jia, G., Aroor, A. R., Jia, C. \& Sowers, J. R. Endothelial cell senescence in aging-related vascular dysfunction. Biochim Biophys Acta Mol Basis Dis 1865, 1802-1809 (2019).

87. Nortley, R. et al. Amyloid $\beta$ oligomers constrict human capillaries in Alzheimer's disease via signaling to pericytes. Science 365, (2019). 
88. Nikolakopoulou, A. M. et al. Pericyte loss leads to circulatory failure and pleiotrophin depletion causing neuron loss. Nat Neurosci 22, 1089-1098 (2019).

89. Sengillo, J. D. et al. Deficiency in mural vascular cells coincides with blood-brain barrier disruption in Alzheimer's disease. Brain Pathol 23, 303-310 (2013).

90. van Veluw, S. J. et al. Detection, risk factors, and functional consequences of cerebral microinfarcts. Lancet Neurol 16, 730-740 (2017).

91. Greenberg, S. M. et al. Cerebral amyloid angiopathy and Alzheimer disease - one peptide, two pathways. Nat Rev Neurol 16, 30-42 (2020).

92. Alarcon-Martinez, L. et al. Interpericyte tunnelling nanotubes regulate neurovascular coupling. Nature 585, 91-95 (2020).

93. Hall, C. N. et al. Capillary pericytes regulate cerebral blood flow in health and disease. Nature 508, 55-60 (2014).

94. Blanchard, J. W. et al. Reconstruction of the human blood-brain barrier in vitro reveals a pathogenic mechanism of APOE4 in pericytes. Nature Medicine 1-12 (2020).

95. Lendahl, U., Nilsson, P. \& Betsholtz, C. Emerging links between cerebrovascular and neurodegenerative diseases-a special role for pericytes. EMBO Rep 20, e48070 (2019).

96. Kalaria, R. N. \& Hedera, P. Differential degeneration of the cerebral microvasculature in Alzheimer's disease. Neuroreport 6, 477-480 (1995).

97. Baloyannis, S. J. \& Baloyannis, I. S. The vascular factor in Alzheimer's disease: a study in Golgi technique and electron microscopy. J Neurol Sci 322, 117-121 (2012).

98. Cuchillo-lbañez, I. et al. The $\beta$-amyloid peptide compromises Reelin signaling in Alzheimer's disease. Sci Rep 6, 31646 (2016).

99. Durakoglugil, M. S., Chen, Y., White, C. L., Kavalali, E. T. \& Herz, J. Reelin signaling antagonizes beta-amyloid at the synapse. Proc Natl Acad Sci U S A 106, 15938-15943 (2009).

100. Rossi, D. et al. Reelin reverts biochemical, physiological and cognitive alterations in mouse models of Tauopathy. Prog Neurobiol 186, 101743 (2020).

101. Botella-López, A. et al. Reelin expression and glycosylation patterns are altered in Alzheimer's disease. Proc Natl Acad Sci U S A 103, 5573-5578 (2006).

102. Herring, A. et al. Reelin depletion is an early phenomenon of Alzheimer's pathology. $J$ Alzheimers Dis 30, 963-979 (2012).

103. Millar, P. R. et al. Evaluating resting-state BOLD variability in relation to biomarkers of preclinical Alzheimer's disease. Neurobiol Aging 96, 233-245 (2020).

104. Grady, C. L. \& Garrett, D. D. Understanding variability in the BOLD signal and why it matters for aging. Brain Imaging Behav 8, 274-283 (2014).

105. Göttler, J. et al. Reduced blood oxygenation level dependent connectivity is related to hypoperfusion in Alzheimer's disease. J Cereb Blood Flow Metab 39, 1314-1325 (2019).

106. Brunskill, E. W. \& Potter, S. S. Pathogenic pathways are activated in each major cell type of the glomerulus in the Cd2ap mutant mouse model of focal segmental glomerulosclerosis. BMC Nephrol 16, 71 (2015).

107. Kim, J. M. et al. CD2-associated protein haploinsufficiency is linked to glomerular disease susceptibility. Science 300, 1298-1300 (2003).

108. Sweeney, M. D. et al. Vascular dysfunction-The disregarded partner of Alzheimer's disease. Alzheimers Dement 15, 158-167 (2019).

109. Viggiano, D. et al. Mechanisms of cognitive dysfunction in CKD. Nature Reviews Nephrology 1-18 (2020).

110. Chen, T. K., Knicely, D. H. \& Grams, M. E. Chronic Kidney Disease Diagnosis and Management: A Review. JAMA 322, 1294-1304 (2019).

111. Kivipelto, M., Mangialasche, F. \& Ngandu, T. Lifestyle interventions to prevent cognitive impairment, dementia and Alzheimer disease. Nat Rev Neurol 14, 653-666 (2018). 
112. Buchman, A. S. et al. Kidney function is associated with the rate of cognitive decline in the elderly. Neurology 73, 920-927 (2009).

113. Kunkle, B. W. et al. Novel Alzheimer Disease Risk Loci and Pathways in African American Individuals Using the African Genome Resources Panel: A Meta-analysis. JAMA Neurol (2020).

114. Elias, M. F., Dore, G. A. \& Davey, A. in Brain, Stroke and Kidney $17942-57$ (Karger Publishers, 2013).

115. Zammit, A. R., Katz, M. J., Bitzer, M. \& Lipton, R. B. Cognitive impairment and dementia in older adults with chronic kidney disease: a review. Alzheimer disease and associated disorders 30, 357 (2016).

116. Förster, E. et al. Reelin, Disabled 1, and beta 1 integrins are required for the formation of the radial glial scaffold in the hippocampus. Proc Natl Acad Sci U S A 99, 13178-13183 (2002).

117. Jiang, Y. et al. Ndel1 and Reelin Maintain Postnatal CA1 Hippocampus Integrity. $J$ Neurosci 36, 6538-6552 (2016).

118. Weeber, E. J. et al. Reelin and ApoE receptors cooperate to enhance hippocampal synaptic plasticity and learning. J Biol Chem 277, 39944-39952 (2002).

119. Tran, C. H. \& Gordon, G. R. Acute two-photon imaging of the neurovascular unit in the cortex of active mice. Front Cell Neurosci 9, 11 (2015).

120. Rosenegger, D. G., Tran, C. H., LeDue, J., Zhou, N. \& Gordon, G. R. A high performance, cost-effective, open-source microscope for scanning two-photon microscopy that is modular and readily adaptable. PLoS One 9, e110475 (2014).

121. Pologruto, T. A., Sabatini, B. L. \& Svoboda, K. Scanlmage: flexible software for operating laser scanning microscopes. Biomed Eng Online 2, 13 (2003).

122. Thirion, J. P. Image matching as a diffusion process: an analogy with Maxwell's demons. Med Image Anal 2, 243-260 (1998).

123. Vercauteren, T., Pennec, X., Perchant, A. \& Ayache, N. Diffeomorphic demons: efficient non-parametric image registration. Neuroimage 45, S61-72 (2009).

124. Mishra, R. C. et al. Alpha 1 -adrenergic stimulation selectively enhances endotheliummediated vasodilation in rat cremaster arteries. Physiol Rep 6, e13703 (2018).

125. Bourasset, F. et al. Reduction of the cerebrovascular volume in a transgenic mouse model of Alzheimer's disease. Neuropharmacology 56, 808-813 (2009).

126. Park, S. K. et al. Par-4 links dopamine signaling and depression. Cell 122, 275-287 (2005).

127. Rubinson, D. A. et al. A lentivirus-based system to functionally silence genes in primary mammalian cells, stem cells and transgenic mice by RNA interference. Nat Genet 33, 401406 (2003).

128. Pelossof, R. et al. Prediction of potent shRNAs with a sequential classification algorithm. Nat Biotechnol 35, 350-353 (2017).

129. Challis, R. C. et al. Systemic AAV vectors for widespread and targeted gene delivery in rodents. Nat Protoc 14, 379-414 (2019).

130. Hashem, M., Zhang, Q., Wu, Y., Johnson, T. W. \& Dunn, J. F. Using a multimodal nearinfrared spectroscopy and MRI to quantify gray matter metabolic rate for oxygen: A hypothermia validation study. Neuroimage 206, 116315 (2020).

131. Johnson, T. W. Measurement of brain oxygenation and metabolism in a mouse model of multiple sclerosis. (2017).

132. Buxton, R. B. Quantifying CBF with arterial spin labeling. Journal of Magnetic Resonance Imaging: An Official Journal of the International Society for Magnetic Resonance in Medicine 22, 723-726 (2005).

133. Pekar, J. et al. Perfusion imaging with compensation for asymmetric magnetization transfer effects. Magnetic resonance in medicine 35, 70-79 (1996). 
134. Herscovitch, P. \& Raichle, M. E. What is the correct value for the brain-blood partition coefficient for water. Journal of Cerebral Blood Flow \& Metabolism 5, 65-69 (1985).

135. Leithner, C. et al. Determination of the brain-blood partition coefficient for water in mice using MRI. Journal of Cerebral Blood Flow \& Metabolism 30, 1821-1824 (2010).

136. Bennett, D. A. Postmortem indices linking risk factors to cognition: results from the Religious Order Study and the Memory and Aging Project. Alzheimer Dis Assoc Disord 20, S63-8 (2006).

137. Tremblay, C. et al. Biochemical characterization of Abeta and tau pathologies in mild cognitive impairment and Alzheimer's disease. J Alzheimers Dis 12, 377-390 (2007).

138. Tremblay, C., St-Amour, I., Schneider, J., Bennett, D. A. \& Calon, F. Accumulation of transactive response DNA binding protein 43 in mild cognitive impairment and Alzheimer disease. J Neuropathol Exp Neurol 70, 788-798 (2011).

139. Bennett, D. A. et al. Natural history of mild cognitive impairment in older persons. Neurology 59, 198-205 (2002).

140. Bennett, D. A., Wilson, R. S., Boyle, P. A., Buchman, A. S. \& Schneider, J. A. Relation of neuropathology to cognition in persons without cognitive impairment. Ann Neurol 72, 599609 (2012).

141. Wilson, R. S. et al. Individual differences in rates of change in cognitive abilities of older persons. Psychol Aging 17, 179-193 (2002).

142. Arvanitakis, Z. et al. Statins, incident Alzheimer disease, change in cognitive function, and neuropathology. Neurology 70, 1795-1802 (2008).

143. Arvanitakis, Z., Capuano, A. W., Leurgans, S. E., Bennett, D. A. \& Schneider, J. A. Relation of cerebral vessel disease to Alzheimer's disease dementia and cognitive function in elderly people: a cross-sectional study. Lancet Neurol 15, 934-943 (2016).

144. Montine, T. J. et al. National Institute on Aging-Alzheimer's Association guidelines for the neuropathologic assessment of Alzheimer's disease: a practical approach. Acta Neuropathol 123, 1-11 (2012).

145. Thal, D. R., Rüb, U., Orantes, M. \& Braak, H. Phases of A beta-deposition in the human brain and its relevance for the development of AD. Neurology 58, 1791-1800 (2002).

146. Braak, H. \& Braak, E. Neuropathological stageing of Alzheimer-related changes. Acta Neuropathol 82, 239-259 (1991).

147. Mirra, S. S. et al. The Consortium to Establish a Registry for Alzheimer's Disease (CERAD). Part II. Standardization of the neuropathologic assessment of Alzheimer's disease. Neurology 41, 479-486 (1991). 


\section{Acknowledgments}

This work was supported by Canadian Institutes of Health and Research (CIHR, F.C. and M.D.N, grant no: 10021444), a postdoctoral scholarship from CIHR and Alberta Innovates Health solution (M.V.), The Religious Orders Study is supported by the National institute on Aging (P30AG10161, R01AG15819, D.A.B.). Special thanks to Dr Amir Sanati Nezhad for the hCMEC/D3 cell line, Dr Frank Visser for the preparation of the viral vectors and Dr Ciaran Murphy-Royal for his comments on the manuscript.

\section{Author contributions}

M.V. designed the study, performed and analyzed the cell culture and awake two photon experiments, analyzed the human data, wrote the manuscript and build the figures, C.G. set up the protocol for the culture of the primary mouse BECs and helped with the cell culture experiments. A.I. trained M.V., helped with the experimental design and data analysis for the awake two-photon experiments. P.B. extracted the protein from the isolated brain vessels, and performed the western blot for the human samples. R.C.M. designed, performed and analyzed the pressure myography experiments. G.P. assisted M.V at the beginning of the two-photon experiments. C.B. tested the siRNA for the cell experiment and designed the protocol for the primary BECs cultures. Y.J. prepared the Reelin solution for the cell culture experiments and did the immunofluorescence on the mouse tissue. L.R. performed the immunohistochemistry with the human tissue. C.T. extracted proteins from the human samples and performed the ELISA and western blot to characterize the human cortex samples. W.N. wrote the script for the analysis of the awake two-photon experiments with the AAV-BR1 mice. A.P.B. supervised R.C.M. for the pressure myography experiments. D.A.B., provided the human samples and clinical data from the Religious Orders Study participants, and critically reviewed the manuscript. G.R.G. supervised G.P. and A.I. for the awake two-photon experiments. F.C. provided the 
human data and supervised P.B., L.R. and C.T. for the human experiments. M.D.N. supervised M.V. C.G., Y.J. and C.B., designed the experiments and wrote the manuscript.

\section{Competing interests}

The authors declare no competing interests. 
bioRxiv preprint doi: https://doi.org/10.1101/2020.12.10.419598; this version posted December 14, 2020. The copyright holder for this preprint (which was not certified by peer review) is the author/funder. All rights reserved. No reuse allowed without permission.

\section{Figures}




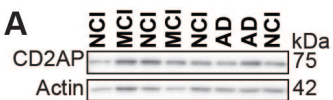
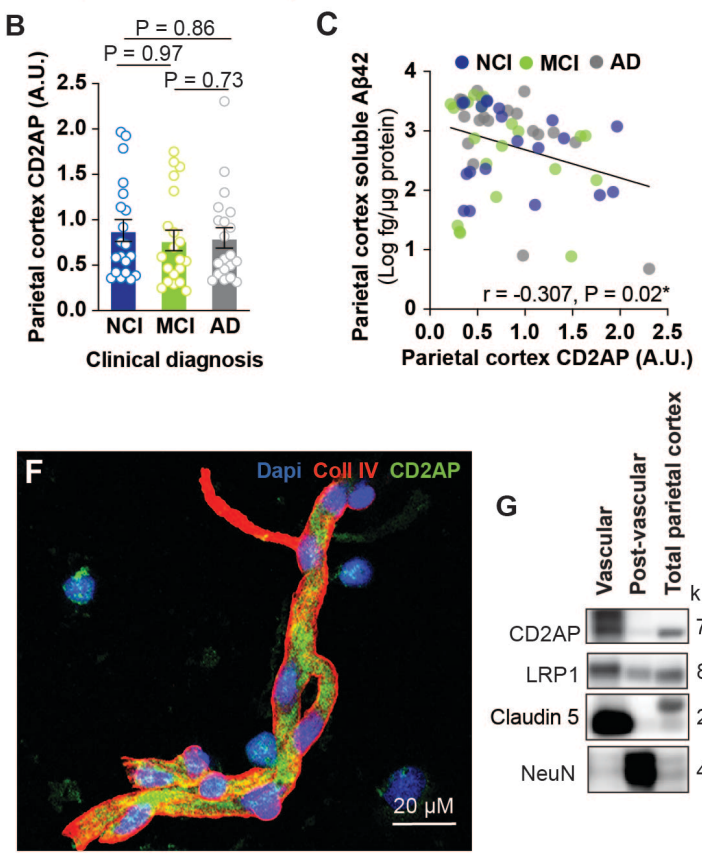

D

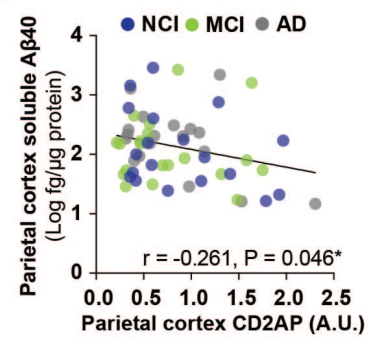

E

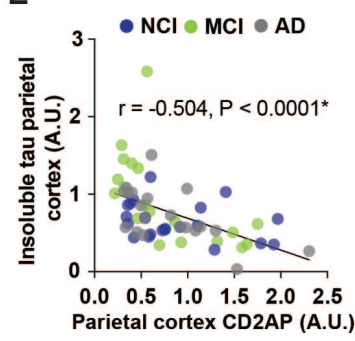

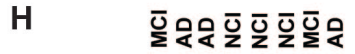
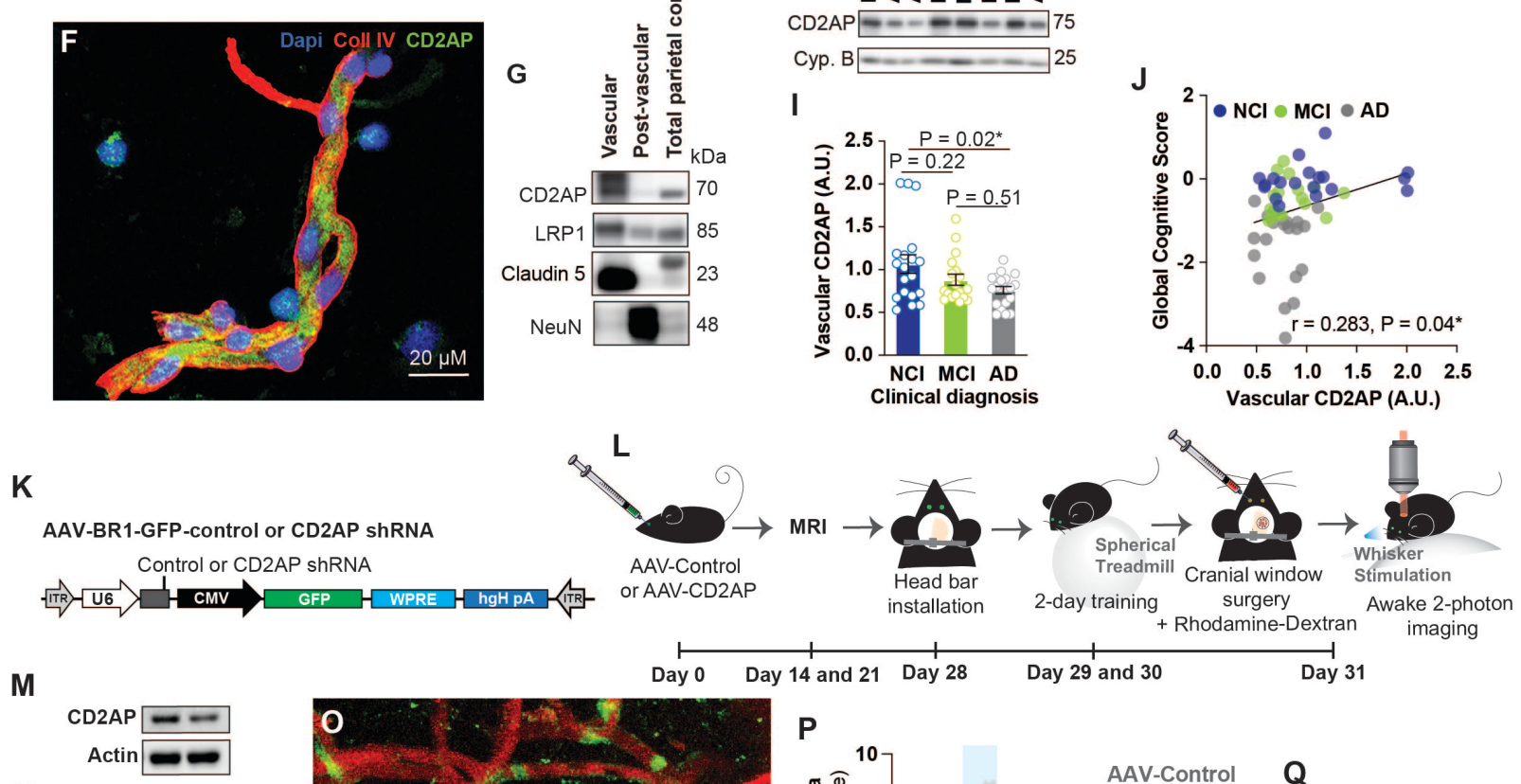

$\mathbf{N}$

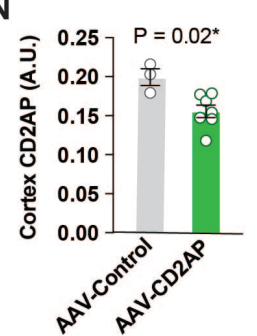

$\mathbf{R}$

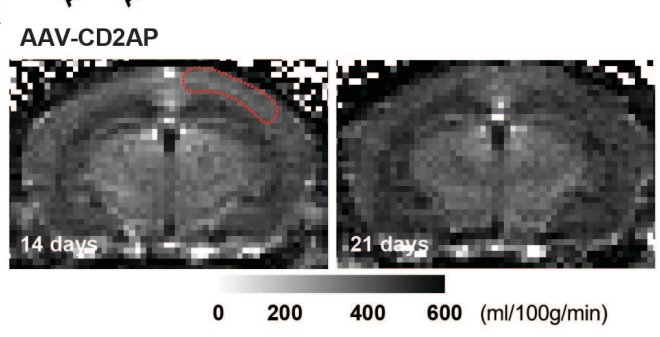

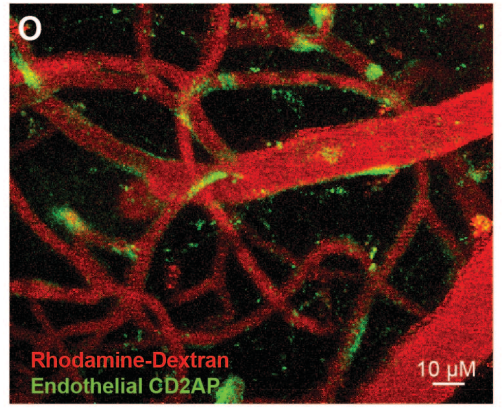

$S$

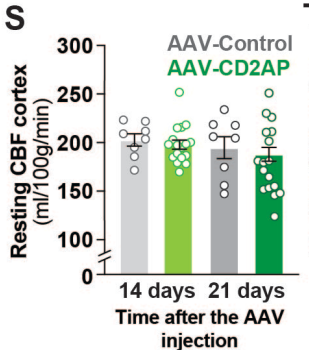

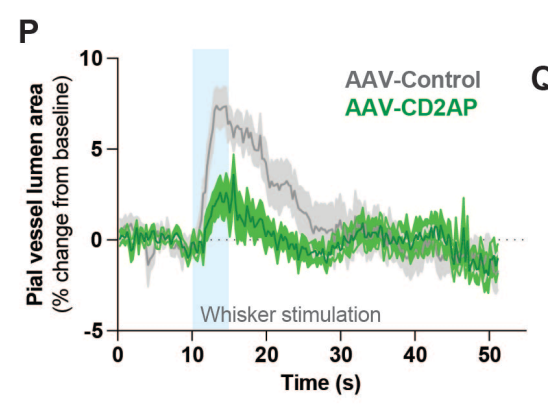

T

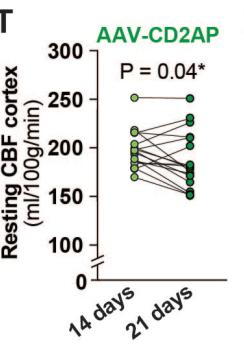

Q
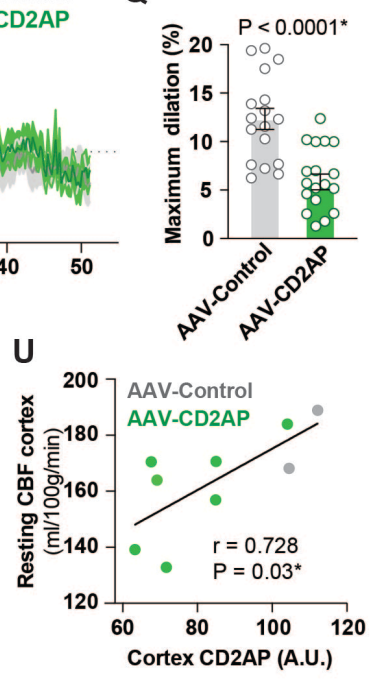

Figure 1: Lower levels of brain endothelial CD2AP are associated with cognitive dysfunction in $A D$ and deregulate functional hyperemia 
A) Representative western blot bands of CD2AP in total parietal cortices from volunteers of the Religious Order Study cohort.

B) CD2AP levels in total parietal cortex homogenates from volunteers classified according to clinical diagnosis: no cognitive impairment $(\mathrm{NCl}, \mathrm{n}=20)$, mild cognitive impairments $(\mathrm{MCl}, \mathrm{n}=$ 20 ) and Alzheimer's disease $(A D, n=20)$. One-way ANOVA, $F(2,57)=0.3039, P=0.7391$, followed by Tukey's post hoc analysis.

(C-E) Correlations between parietal cortex CD2AP and C) soluble A $\beta 42$, D) soluble A $\beta 40$ and E) insoluble total tau. Pearson $r$. The association remained significant after adjustment for age of death and education.

F) Immunohistochemistry for CD2AP (green), Collagen IV (Coll IV, red) and Dapi (blue) in isolated brain vessels.

G) Representative western blot of CD2AP, LRP1 (LDL Receptor Related Protein 1), Claudin 5 and NeuN (Neuronal nuclei) in human brain vascular and post vascular fraction compared to total parietal cortex homogenates.

$\mathrm{H})$ Representative western blot bands of CD2AP in isolated human brain vessels from volunteers of the Religious Order Study cohort.

I) CD2AP levels in volunteers classified according to clinical diagnosis: no cognitive impairment $(\mathrm{NCl}, \mathrm{n}=19)$, mild cognitive impairments $(\mathrm{MCl}, \mathrm{n}=18)$ and Alzheimer's disease (AD, $\mathrm{n}=19)$. One-way ANOVA, $F(2,53)=3.636, P=0.0233$, followed by Tukey's post hoc analysis.

J) Correlation between the levels of vascular CD2AP and the global cognitive score. Pearson $r$. The association remained significant after adjustment for age of death and education or plaques and tangles.

K) Representation of the viral vector construct.

L) Timeline of the in vivo experiments.

M) Representative western blot bands for the levels of CD2AP in cortices of mice infected with AAV-control and AAV-CD2AP.

$\mathrm{N}$ ) CD2AP levels in mouse cortices 21 days after the AAV injection (AAV-control, $n=3, A A V$ CD2AP, $n=7$ ). Unpaired Student t-test, $t=3.056$, $d f=8$.

O) Z-stack reconstructed image of the vasculature in the barrel cortex of an AAV-BR1 mouse injected with Rhodamine-Dextran and visualized with a two-photon microscope (Green = GFP, Red $=$ Rhodamine-Dextran).

$P)$ Change in pial vessel luminal area in response to whisker stimulation (5 s) in AAV-Control (n $=7)$ and AAV-CD2AP $(n=8)$ mice.

Q) Maximal pial vessel dilation after whisker stimulation (Unpaired Student t-test, $\mathrm{t}=4.860$, $\mathrm{df}=$ 34, each dot represents an individual stimulation, AAV-Control: $n=17$, AAV-CD2AP: $n=18$ ).

$\mathrm{R})$ Representative images of brain perfusion in AAV-CD2AP mice taken during magnetic resonance imaging (MRI). The red line represents the zone analyzed for resting cerebral blood flow (CBF).

S) Resting cortex CBF in mice injected with AAV (AAV-control, $n=8, A A V-C D 2 A P, n=17$ ). Two-way ANOVA, Time x genotype $(F(1,23)=0.02030, P=0.8879)$, Time $(F(1,23)=3.935, P=$ $0.0593)$, Genotype $(F(1,23)=0.3153, P=0.5799)$.

T) Resting cortex CBF in AAV-CD2AP mice 14 and 21 days after viral vector injection. Paired Student $\mathrm{t}$-test, $\mathrm{t}=2.214, \mathrm{df}=16$.

$\mathrm{U})$ Correlation between resting cortex CBF and CD2AP levels, 21 days after AAV injections.

Pearson r.

The levels of CD2AP in the parietal cortices and brain vascular fractions are normalized to the levels of actin and cyclophilin $B$, respectively.

For gel source data, see Supplementary File. 
A

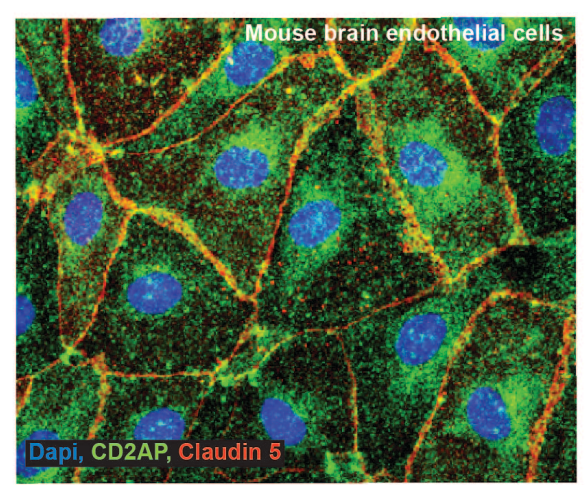

E

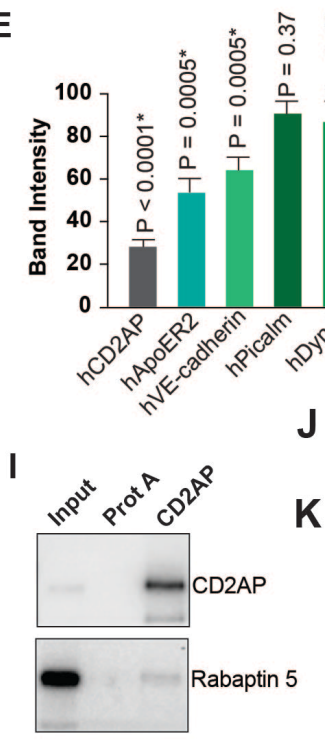

B

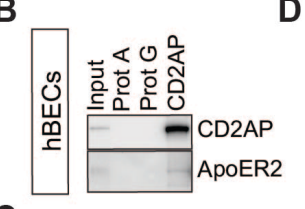

C

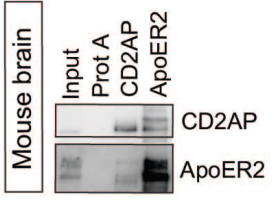

D

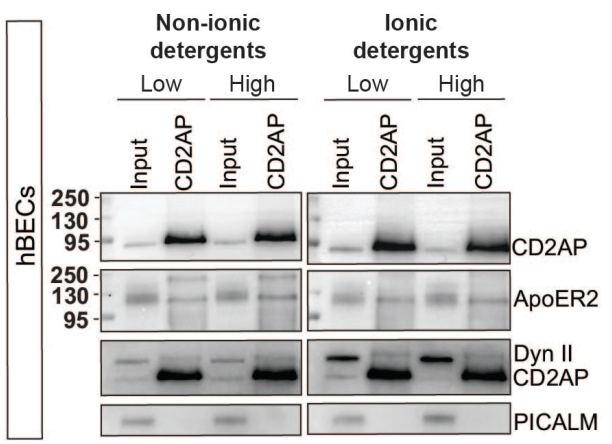

Ctrl KD

G

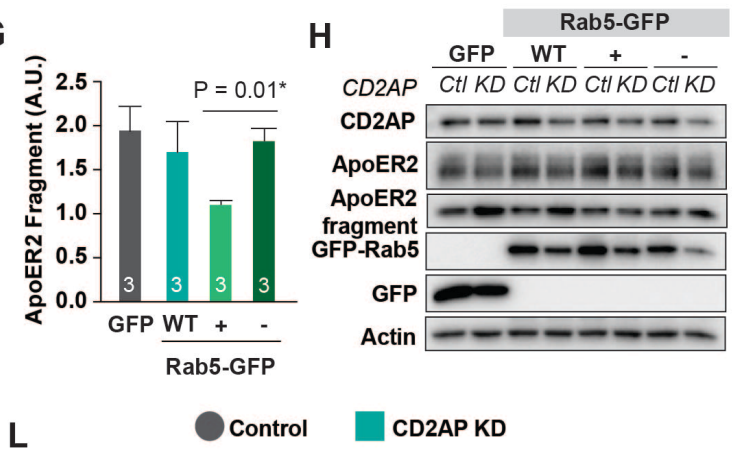

J $C D 2 A P \overline{C t / K D C t / K D} \overline{C t / K D} C t / K D$ CD2AP $=-1=-2=$

L

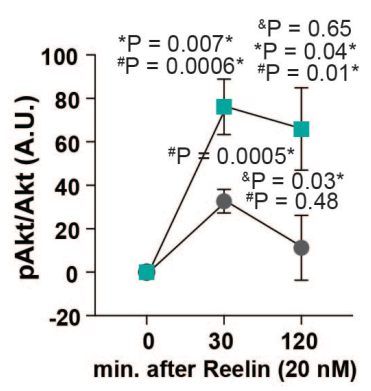

Figure 2: CD2AP regulates Reelin-mediated ApoER2 signaling and levels in brain endothelial cells

A) Immunohistochemistry for CD2AP in mouse primary BECs.

B-D) Co-immunoprecipitation of CD2AP with ApoER2 in B) human brain endothelial cell line (hCMEC/D3, hBECs), C) adult mouse brain and D) hBECs under ionic and non-ionic detergent. E) Effect of CD2AP depletion on the levels of specific proteins in hBECs ( $n=9$ wells, One sample t-test compared to 100, CD2AP: $\mathrm{t}=13.85$, ApoER2: $\mathrm{t}=5.567$, VE-Cadherin: $\mathrm{t}=5.662$, PICALM: $t=0.9413$, Dyn $2: t=1.887, d f=8)$ and mouse primary brain endothelial cells (mBECs) ( $n=9$ wells, One sample t-test compared to 100, CD2AP: $t=21.04$, ApoER2: $t=$ 3.751, Picalm: $t=0.009, \mathrm{df}=8$ ). Data are presented as a $\%$ of the mean of cells transfected with control siRNA.

F) Representative western blots from control and CD2AP KD hBECs cells.

G) Levels of ApoER2 in CD2AP KD hBECs cells transfected with WT, overactive or inactive Rab5. Unpaired Student t-test between Rab5-GFP + and Rab5-GFP -, $P=0.0148, t=4.107$, df $=4$.

H) Representative western blots from control and CD2AP KD hBECs cells transfected with WT, overactive or inactive Rab5.

I) Rabaptin-5 co-immunoprecipitates with CD2AP in adult mouse brain. 
J) Representative western blot from control and CD2AP KD hBECs cells treated with DMSO of $\gamma$-secretase inhibitor (DAPT).

K) CD2AP and ApoER2 levels in hBECs cells depleted of CD2AP treated with $\gamma$-secretase inhibitor. Unpaired Student t-test. ApoER2, $P=0.0252, t=3.489, d f=4$.CD2AP, $P=0.0533, t=$ 2.715, df $=4$.

L) ApoER2 and pAkt/Akt levels in hBECs in control or CD2AP KD cells treated with Reelin (20 $\mathrm{nM})$ or control solution for $30 \mathrm{~min}$ and $120 \mathrm{~min}$. Control and CD2AP KD $30 \mathrm{~min}(\mathrm{n}=8)$, Control and CD2AP KD 120 min $(n=7)$. ${ }^{*}$ KD vs Ctrl, ApoER2 (30 min: Unpaired Student $t$-test, $\mathrm{t}=$ 4,757, df = 14, 120 min: Unpaired Student t-test, $t=4,869$, df = 12), pAkt/Akt (30 min: Unpaired Student t-test with Welch's correction: $\mathrm{t}=3.127, \mathrm{df}=9,120 \mathrm{~min}$ : Unpaired Student $\mathrm{t}$-test, $\mathrm{t}=$ 2.268, df = 12), " $30 \mathrm{~min}$ vs $120 \mathrm{~min}$, ApoER2 (Control: Unpaired Student t-test, $t=2.588 \mathrm{df}=13$, CD2AP KD: Unpaired Student t-test, $t=1.052$, df $=13$ ), pAkt/Akt (Control: Unpaired Student ttest with Welch's correction, $\mathrm{t}=1.351, \mathrm{df}=7.5, \mathrm{CD} 2 \mathrm{AP}$ KD: Unpaired Student $\mathrm{t}$-test, $\mathrm{t}=0.4577$, $\mathrm{df}=1$ ). " Change vs baseline, one-sample t-test compare to zero, ApoER2 (Control $30 \mathrm{~min}: \mathrm{t}=$ 1.122, $\mathrm{df}=7$, Control $120 \mathrm{~min}: \mathrm{t}=3.808, \mathrm{df}=8$, CD2AP KD $30 \mathrm{~min}: \mathrm{t}=5.912, \mathrm{df}=7, \mathrm{CD} 2 \mathrm{AP} \mathrm{KD}$ $120 \mathrm{~min} t=3.036, \mathrm{df}=6$ ), pAkt/Akt (Control $30 \mathrm{~min}: \mathrm{t}=6.015, \mathrm{df}=7$, Control $120 \mathrm{~min}: \mathrm{t}=$ 0.7566, $d f=6$, CD2AP KD $30 \mathrm{~min}: \mathrm{t}=5.958, \mathrm{df}=7$, CD2AP KD $120 \mathrm{~min} . \mathrm{t}=3.483, \mathrm{df}=6$ ).

CD2AP: CD2-associated protein, ApoER2: apolipoprotein E receptor 2, KD: knockdown, DMSO: Dimethylsulfoxyde. DAPT: N-[N-(3,5-Difluorophenacetyl)- L -alanyl]-S-phenylglycine t-butyl Ester. GFP: Green Fluorescent Protein. Rab5: Ras-related protein 5. Uncropped gels can be found in Supplementary File. 

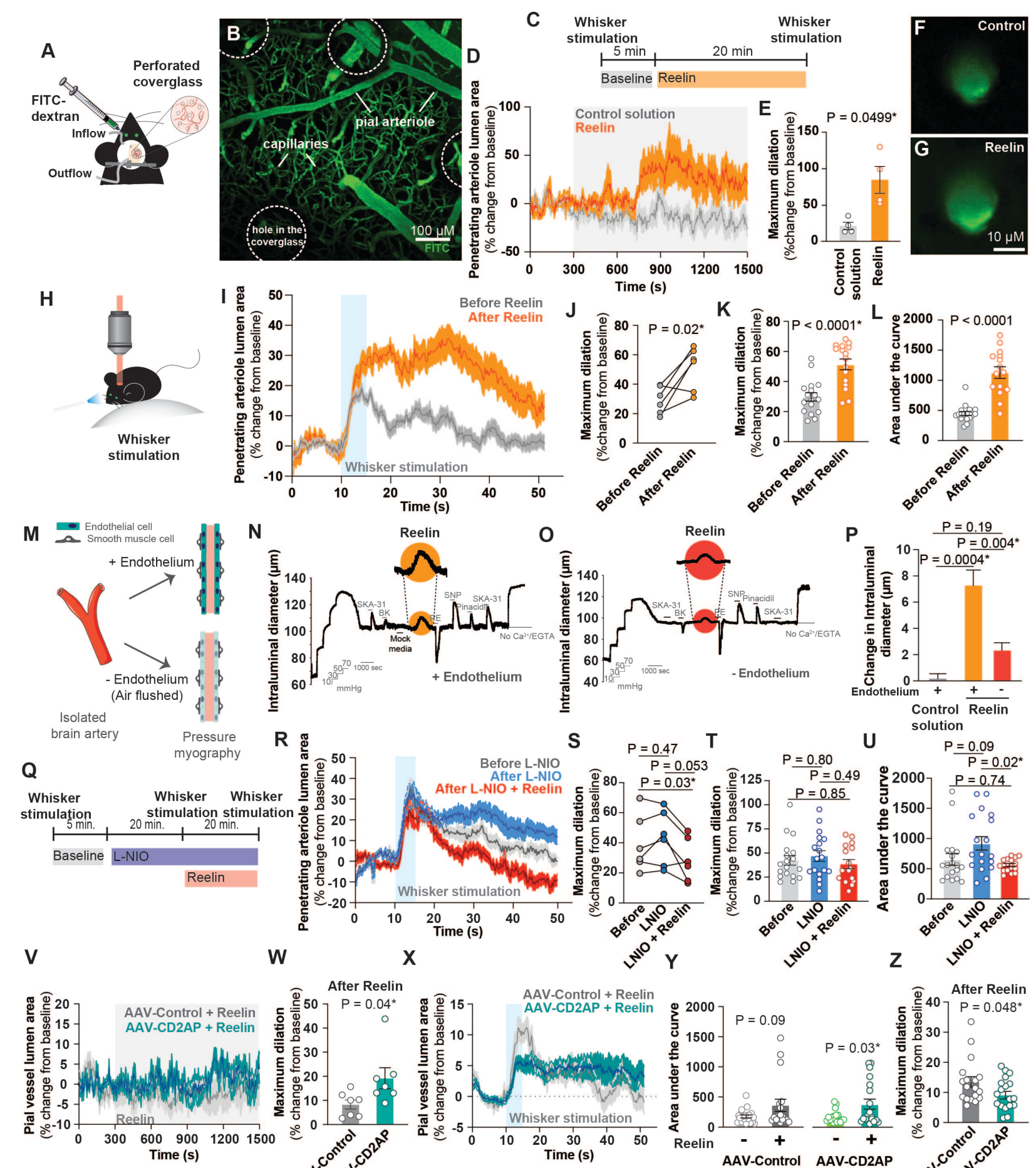

$P=0.09$
$P=0.02$

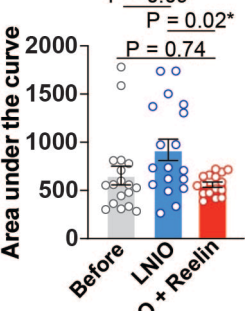
W $\quad \mathrm{X}$
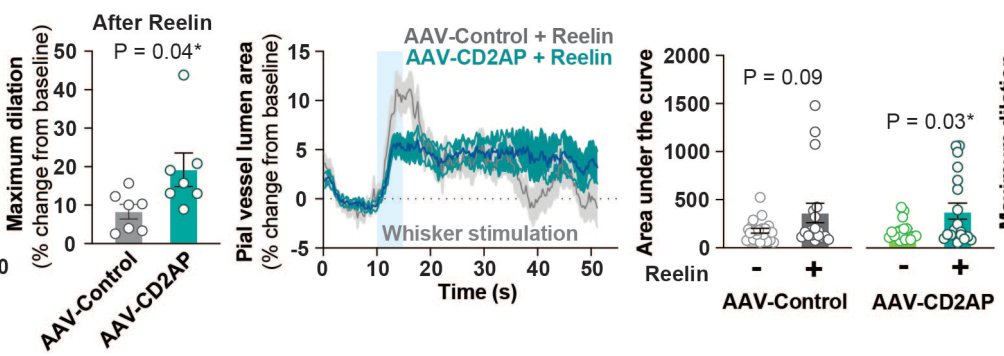

$2 \quad 2$

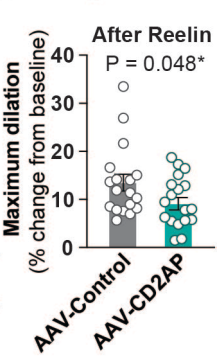

Figure 3: The effects of Reelin on vessel dilation and functional hyperemia are modulated by brain endothelial CD2AP

A) Illustration representing the awake mice experimentation setup.

B) Z-stack reconstructed image of the barrel cortex in a C57BL6 mouse mounted with a cover glass with holes and depicting FITC-dextran (green) vessels.

C) Timeline of the drug application during the in vivo experiments. 
D) Effect of control solution or Reelin $(1 \mu \mathrm{M})$ on penetrating arterioles transversal luminal area $(\mathrm{n}$ $=4$ mice).

E) Maximum dilation of a penetrating arteriole in response to control solution or Reelin (Paired Student t-test, $\mathrm{t}=3.185, \mathrm{df}=3)$.

Representative image (Green: FITC-dextran) of a penetrating arteriole after superfusion with F) control solution or G) Reelin.

$\mathrm{H})$ Illustration of the whisker stimulation during the awake two-photon imaging.

I) Penetrating arteriole transversal luminal area in response to whisker stimulation ( $5 \mathrm{~s}$ ) before and after Reelin application ( $n=6$ mice, each dot represents the average of 3 stimulations).

Maximum dilation of a penetrating arteriole after whisker stimulation I) per animal (Paired Student t-test, $\mathrm{t}=3.286$, $\mathrm{df}=5$ ) or $\mathrm{K}$ ) for each stimulation (Before $\mathrm{n}=17$ stimulations, After $\mathrm{n}=$ 16 stimulations, Unpaired Student t-test, $\mathrm{t}=4.807$, $\mathrm{df}=31$ ).

$\mathrm{L}$ ) Overall response to whisker stimulation (Unpaired Student $\mathrm{t}$-test with Welch's correction, $\mathrm{t}=$ 6.643 , df = 17.12).

M) Illustration representing the pressure myography experiment.

$\mathrm{N}-\mathrm{O}$ ) Representative tracings displaying acute vasoactive responses to endothelium $\mathrm{N}$ ) dependent and $\mathrm{O}$ ) independent agents in single cannulated cerebral arteries from C57BL6 mice. SKA-31 (3 and $15 \mu \mathrm{M})$, bradykinin (BK, $0.5 \mu \mathrm{M})$, control solution (volume matched with Reelin), Reelin $(1 \mu \mathrm{M})$, and the endothelium-independent agent phenylephrine (PE, $250 \mathrm{nM})$, sodium nitroprusside (SNP, $10 \mu \mathrm{M})$ and pinacidil $(10 \mu \mathrm{M})$. Horizontal bars above the tracing indicate the treatment with each agent.

$P)$ Changes in intraluminal diameter of cerebral arteries triggered by the indicated vasoactive agents $(n=4$ mice, One-way ANOVA, $P=0.0004, F(2,9)=20,92$ followed by Tuckey's post hoc analysis).

Q) Illustration representing the timing for the whisker stimulation and drugs application during the awake two-photon imaging experiment.

$\mathrm{R})$ Changes of penetrating arteriole transversal luminal area in response to whisker stimulation ( $5 \mathrm{~s}, 4 \mathrm{~Hz}$ ) before and after L-NIO and L-NIO + Reelin application.

$\mathrm{S})$ Maximum dilation during whisker stimulation of penetrating arterioles at baseline, after L-NIO and after L-NIO + Reelin (Repeated measure ANOVA, F $(1.452,7.258$ ) = 8.842, P = 0.0148, followed by Tuckey's post hoc analysis, each dot represents the average of 3 stimulations).

T) Maximum dilation during whisker stimulation of penetrating arterioles at baseline, after L-NIO and after L-NIO + Reelin (One-way ANOVA, F(2,49) = 0.6634, P = 0.5197, each dot represents one stimulation).

U) Overall response to whisker stimulation (One-way ANOVA, $F(2,49)=4.328, P=0.0186$ ).

V) Effects of AAV-Control and AAV-CD2AP on pial vessel luminal area in response to Reelin (1 $\mu \mathrm{M})$ superfusion.

W) Maximal dilation of pial vessels in response to Reelin (Unpaired Student t-test, $t=2.551$, df $=$ 8 , each dot represents one animal).

$X)$ Pial vessel transversal luminal area in response to whisker stimulation $(5 \mathrm{~s}, 4 \mathrm{~Hz})$ in AAVControl and AAV-CD2AP infected mice after Reelin $(1 \mu \mathrm{M})$ superfusion.

Y) Overall whisker stimulation response before and after Reelin superfusion (Unpaired student ttest with Welch's correction, AAV-Control, $\mathrm{t}=1.765, \mathrm{df}=19.10, \mathrm{AAV}-\mathrm{CD} 2 \mathrm{AP}, \mathrm{t}=2.341 \mathrm{df}=$ 20.84, each dot represents one stimulation).

Z) Maximum dilation after whisker stimulation and Reelin superfusion (Unpaired Student $\mathrm{t}$-test, $\mathrm{t}$ $=2.052, \mathrm{df}=35$, each dot represents one stimulation).

Data are presented as mean \pm SEM. 

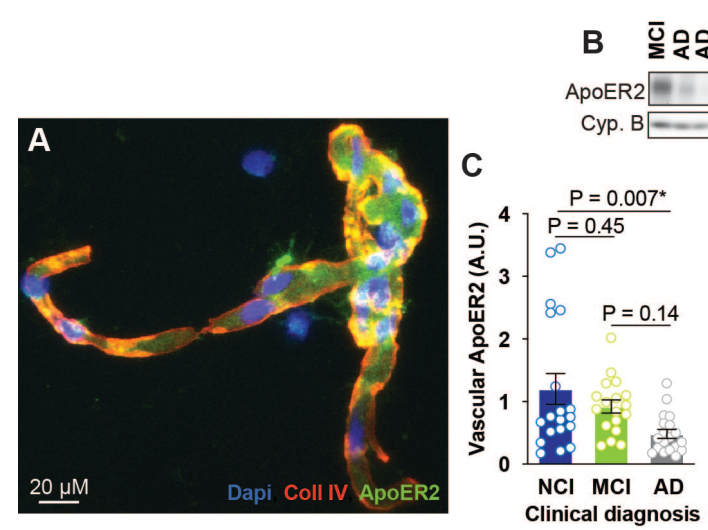
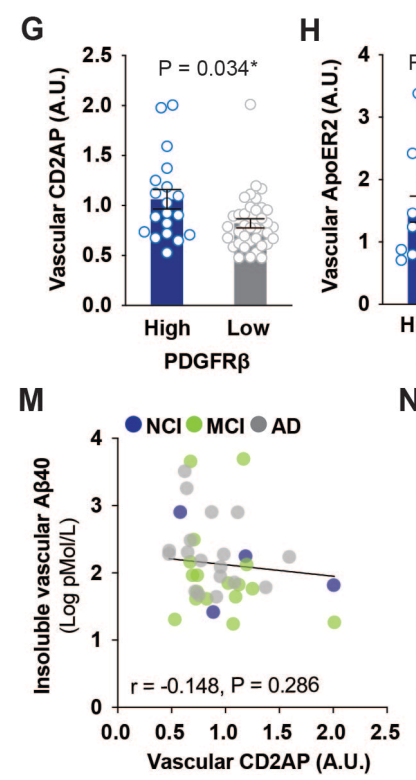

N
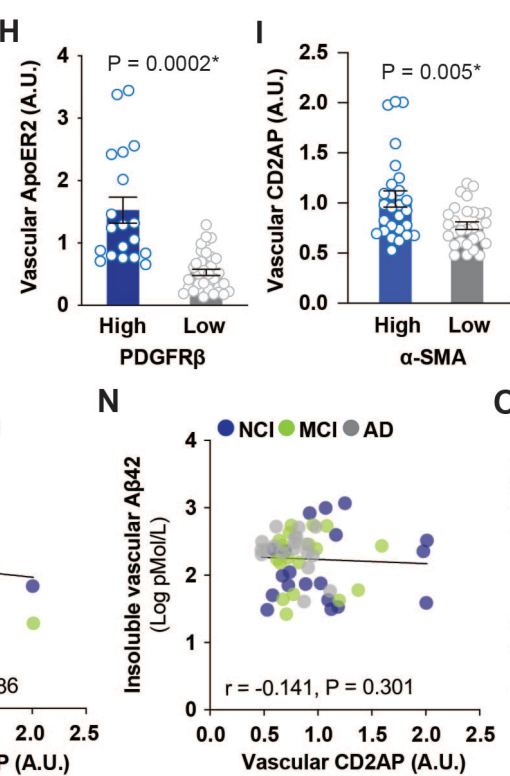
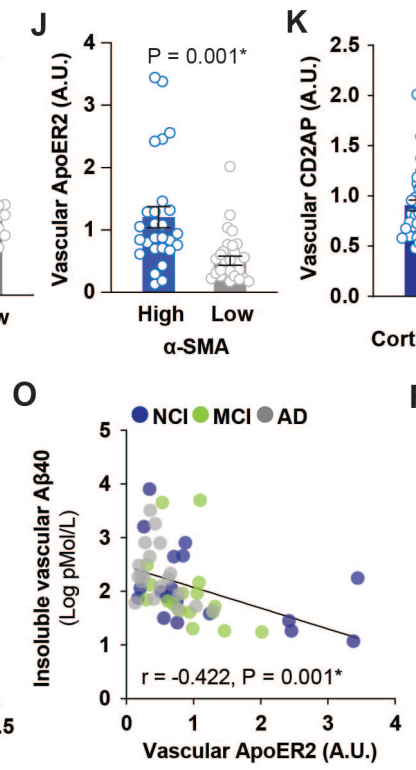

$F$

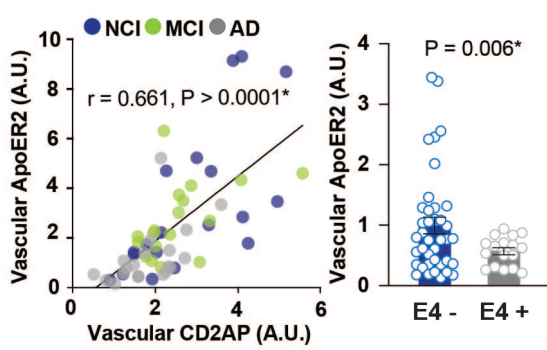

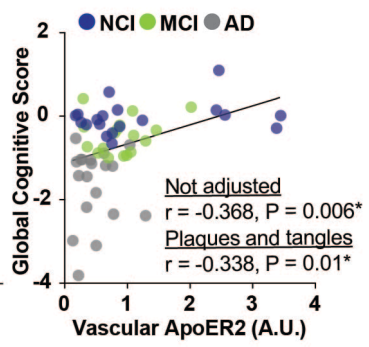

L

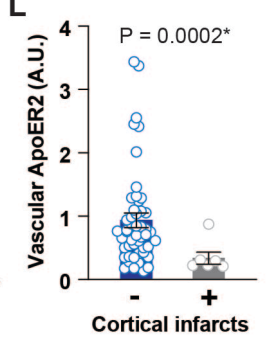

$\mathbf{P}$

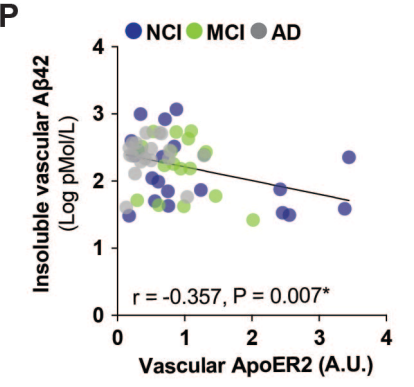

Figure 4: Lower levels of ApoER2 in brain vessels correlate with depletion of vascular CD2AP and are linked to cognitive decline and vascular dysfunction in AD volunteers

A) Immunofluorescent image of a typical brain vessel isolated from a subject of the Religious Order Study cohort stained for ApoER2 (green), Collagen IV (Coll IV, red) and Dapi (blue)

B) Representative western blot bands for ApoER2 in isolated human brain vessels from volunteers of the Religious Order Study cohort.

C) Vascular levels of ApoER2 in volunteers classified according to the clinical diagnosis: $\mathrm{NCl}(\mathrm{n}$ $=19), \mathrm{MCl}(\mathrm{n}=18)$ and $\mathrm{AD}(\mathrm{n}=19)$. One-way ANOVA, $\mathrm{F}(2,53)=5.104, \mathrm{P}=0.0094$, followed by Tukey's post hoc analysis.

D) Correlation between vascular CD2AP and ApoER2. Pearson r. The association remained significant after adjustment for age and the endothelial cell marker (cyclophilin B and Claudin 5).

E) Levels of vascular ApoER2 in APOE4 non-carriers $(E 4-, n=39)$ and carriers $(E 4+, n=17)$.

Unpaired Student t-test with Welch's correction, $\mathrm{t}=2.860$, $\mathrm{df}=49.18$.

F) Correlations between vascular CD2AP and the global cognitive score. Pearson $r$. The association remained significant after adjustment for age of death and education or plaques and tangles.

G) Levels of CD2AP in brain vessels from volunteers classified according to the levels of PDGFR $\beta$ (Low: $n=33$, High: $n=23$, Unpaired Student $t$-test, $t=2.025$, $d f=54$ ). Participants in the Low PDGFR $\beta$ group had PDGFR $\beta$ levels lower than the mean of all volunteers; participants 
in the High PDGFR $\beta$ group had a PDGFR $\beta$ levels higher than the mean of all volunteers. Levels were quantified by western blot.

H) Levels of ApoER2 in brain vessels from volunteers classified according to the levels of PDGFR $\beta$ (Low: $n=33$, High: $n=23$, Unpaired Student t-test, $t=5.578$, $d f=54$ ).

I) Levels of CD2AP in brain vessels from volunteers classified according to the levels of $\alpha$-SMA (Low: $n=29$, High: $n=27$, Unpaired Student $t$-test with Welch's correction. $t=2.971$. $d f=$ 36.76). Participants in the Low $\alpha$-SMA group had a $\alpha$-SMA levels lower than the mean of all volunteers; participants in the High $\alpha$-SMA group had a $\alpha$-SMA levels higher than the mean of all volunteers. Levels were quantified by western blot.

J) Levels of ApoER2 in brain vessels from volunteers classified according to the levels of $\alpha$-SMA (Low: $n=29$, High: $n=27$, Unpaired Student $t$-test with Welch's correction. $t=2.971$. $d f=$ 36.76).

K) Levels of CD2AP in brain vessels from volunteers classified according to the absence (-, $n=49$ ) or presence $(+, n=7)$ of cortical infarcts. Unpaired Student t-test, $t=0.2389, d f=54$.

L) Levels of ApoER2 in brain vessels from volunteers classified according to cortical infarcts (-: $n$ $=49,+: \mathrm{n}=7$, Unpaired Student t-test with Welch's correction, $\mathrm{t}=4.229$, $\mathrm{df}=28.87$ ).

M-N) Correlations between vascular CD2AP and vascular insoluble M) A $\beta 40, N) A \beta 42$. Pearson

r.

O-P) Correlations between vascular ApoER2 and vascular insoluble O) A $\beta 40, P) A \beta 42$. Pearson $r$. The association remained significant after adjustment for age of death and education or plaques and tangles.

The levels of CD2AP and ApoER2 in the brain vascular fraction are normalized to the levels cyclophilin B.

For gel source data see Supplementary File

Data are presented as mean \pm SEM. 